\title{
Off-line simulation inspires insight: A neurodynamics approach to efficient robot task learning
}

\author{
Emanuel Sousa ${ }^{a, *}$, Wolfram Erlhagen ${ }^{b}$, Flora Ferreira ${ }^{b}$, Estela Bicho ${ }^{a}$ \\ ${ }^{a}$ Center Algoritmi, Department of Industrial Electronics, University of Minho, Guimarães, Portugal \\ ${ }^{\mathrm{b}}$ Center of Mathematics, Department of Mathematics and Applications, University of Minho, Guimarães, Portugal
}

\section{A R T I C L E I N F O}

\section{Article history:}

Available online 19 October 2015

\section{Keywords:}

Off-line learning

Adaptive robot

Sequential task

Dynamic neural field

Persistent activity

Social learning

\begin{abstract}
A B S T R A C T
There is currently an increasing demand for robots able to acquire the sequential organization of tasks from social learning interactions with ordinary people. Interactive learning-by-demonstration and communication is a promising research topic in current robotics research. However, the efficient acquisition of generalized task representations that allow the robot to adapt to different users and contexts is a major challenge. In this paper, we present a dynamic neural field (DNF) model that is inspired by the hypothesis that the nervous system uses the off-line re-activation of initial memory traces to incrementally incorporate new information into structured knowledge. To achieve this, the model combines fast activationbased learning to robustly represent sequential information from single task demonstrations with slower, weight-based learning during internal simulations to establish longer-term associations between neural populations representing individual subtasks. The efficiency of the learning process is tested in an assembly paradigm in which the humanoid robot ARoS learns to construct a toy vehicle from its parts. User demonstrations with different serial orders together with the correction of initial prediction errors allow the robot to acquire generalized task knowledge about possible serial orders and the longer term dependencies between subgoals in very few social learning interactions. This success is shown in a joint action scenario in which ARoS uses the newly acquired assembly plan to construct the toy together with a human partner.
\end{abstract}

(C) 2015 Elsevier Ltd. All rights reserved.

\section{Introduction}

Currently, new generations of robots are built that are supposed to interact closely with ordinary people in their working and living environments. These robots have to master a wide variety of everyday tasks that cannot be completely designed in advance by experts as in traditional industrial applications (Schaal, 2007). A major challenge of current robotics research is thus to endow robots with an adaptive, efficient and user-friendly instruction method that would allow ordinary people to teach the robot new tasks in an open-ended manner. Ideally, naïve users may bring their own learning experiences in social interactions with other people to the robotics domain. Learning by observing and imitating others behaviors and their consequences is a powerful social learning mechanism for human-to-human knowledge transfer (Bandura, 1971). It is attractive for the robotics domain as well since learning by observation significantly speeds up

\footnotetext{
* Corresponding author.

E-mail address: esousa@dei.uminho.pt (E. Sousa).
}

skill acquisition compared to individual discovery in potentially dangerous trial-and-error learning. While a full-blown, humanlike social learning capacity for robots still remains a distant goal, major progress has been made over the last decade in various research directions of the programming by demonstration approach (for review papers and collections see e.g., (Billard, Calinon, Dillmann, \& Schaal, 2008; Dautenhahn \& Nehaniv, 2002)). Most robotics experiments thus far have focused on the level of transferring motor skills for object manipulation from human to robot. Some other research started at the more abstract level of learning serial tasks defined by a sequence of subgoals or events in domains like assembly work (Ikeuchi \& Suehiro, 1994), navigation (Nicolescu \& Matarić, 2003) or household manipulation (Pardowitz, Knoop, Dillmann, \& Zöllner, 2007). Recent developments stress the importance of social learning cues such as verbal feedback and communicative gestures to guide the real-time learning process in an incremental manner (Otero, Saunders, Dautenhahn, \& Nehaniv, 2008; Thomaz \& Breazeal, 2008). However, little attention has been paid thus far on the generality of the acquired task knowledge, and the efficiency of the learning process in terms of the number of demonstrations 
needed (Pardowitz et al., 2007; Wu \& Demiris, 2010). To act as an intelligent and flexible co-worker it is not sufficient that the robot memorizes a demonstrated task, such as, for instance assembling a furniture or laying the dinner table, as a simple linear sequence of events. The robot should be able to adapt the serial order of task execution to the preferences of different users, and at the same time should be able to understand and represent the task structure where the achievement of multiple independent subgoals may enable a final outcome. This in turn requires an ability to connect temporally nonadjacent subtasks. Very importantly, since multiple demonstrations of the same task would be time-consuming and annoying for users, the acquisition of generalized task knowledge in very few demonstrations is crucial for user acceptance.

In this paper we present a neurodynamics approach to robot task learning that takes inspiration from a hypothesis about how the nervous system might efficiently consolidate initial memory traces of sequential events into structured knowledge. Converging lines of evidence from computational theories (e.g., connectionist networks McClelland, O'reilly, \& McNaughton, 1995, for review see O'Reilly \& Norman, 2002) and neurophysiological studies (Euston, Tatsuno, \& McNaughton, 2007, for review see Sutherland \& McNaughton, 2000) support the notion of two complementary learning systems that allow distributed neural structures to gradually integrate new input patterns without disturbing previously stored information. A fast system is responsible for the rapid storage of newly demonstrated sequential events. The spontaneous re-activation of these memory traces during "off-line states", in which the neural system is not processing external inputs, facilitates the gradual adjustment of synaptic weights in associative networks of the slow system encoding generalized knowledge of the sequential structure. To model the two complementary learning systems, we apply the theoretical framework of dynamic neural fields (DNFs) that has been proven in the past to provide key processing mechanisms for applications in cognitive modeling (Schöner, 2008) and in cognitive robotics (Erlhagen \& Bicho, 2006). Most importantly, DNFs explain the existence of self-sustained activity in neural populations as the result of reciprocal positive feedback between neighboring neurons (Amari, 1977). Persistent activity has been reported in many areas of higher association cortices and is commonly believed to support a multitude of relevant cognitive functions such as working memory, decision making, and the learning of associations between events separated in time (Curtis \& Lee, 2010; Miller, 2000). The intrinsically stable dynamics of population activity modeled by DNFs allows us not only to implement the short-term maintenance of task-relevant sequential information but also the active rehearsal of this information during off-line learning periods not constrained by the time course of observed events.

To test the learning model in real-world robotics experiments, we adopt a construction task that we have used in previous work to test a DNF architecture for natural and fluent human-robot interactions (HRI) (Bicho, Erlhagen, Louro, \& Costa e Silva, 2011; Bicho, Louro, \& Erlhagen, 2010). The main goal of the present study is to acquire in a social learning situation with human tutors the knowledge about possible serial orders of executing the assembly steps. This shared task knowledge was predefined by the designer in our earlier HRI studies. To this end, one or more human tutors first show the humanoid robot ARoS the assembly work consisting of a series of assembly steps necessary to construct a toy object from its parts. The tutor then provides immediate verbal feedback about predicted next steps when the robot tries to reproduce the serial order from memory. Demonstrations with different serial orders together with the integration of prediction errors in the associative learning process allow the robot to acquire generalized task knowledge in very few social learning interactions. This success is shown in a joint action scenario in which ARoS uses the

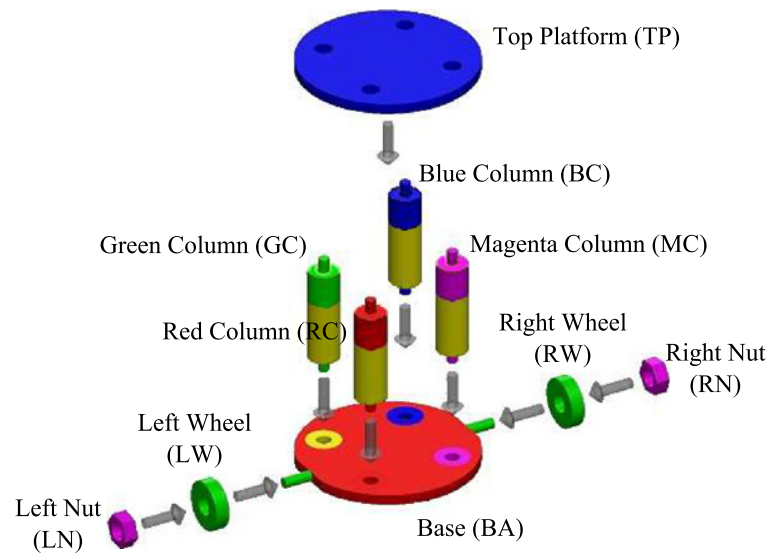

Fig. 1. Assembly plan of the toy vehicle. The columns can be inserted in any order, but the nuts and the top platform can be mounted only if the preceding parts are in place.

newly acquired assembly plan to construct the toy together with a human partner.

The rest of the paper is structured as follows: Section 2 describes the construction task, the learning paradigm and the robotic platform ARoS. Section 3 presents an overview about basic processing principles of the dynamic neural field framework. Section 4 contains the description of the DNF based learning model. Experimental results are presented in Section 5. The paper finishes with a discussion of results and future work in Section 6.

\section{Experimental paradigm and setup}

\subsection{Task description}

By observing a human tutor, the robot has to learn the sequential structure of individual assembly steps necessary to construct a toy vehicle from its components (Fig. 1). The vehicle has a round platform with an axle as its base (BA). On each side, a wheel (RW and LW) has to be attached to the axle and subsequently fixed with a nut (RN and LN, respectively). Subsequently, 4 columns (GC, $\mathrm{BC}, \mathrm{RC}, \mathrm{MC}$ ) identified by their different colors (green, blue, red and magenta, respectively) have to be plugged into holes in the base that match the colors of the columns. The placing of another round object (TP) on top of the columns finishes the task. The columns can be in principle inserted in any order, but TP can only be placed after all columns are in place. This allows us to test the learning of short-term and long-term dependencies between subgoals in relation to the final goal of the assembly work.

\subsection{Learning paradigm}

The experimental paradigm mimics a social learning situation in which the robot ARoS first observes a teacher demonstrating a sequence of assembly steps necessary to build the vehicle, and subsequently shows its acquired task knowledge by trying to execute the assembly work jointly with the teacher. She/he guides the learning of serial order by giving immediate feedback about the correctness of intended assembly steps communicated by the robot and by re-demonstrating the sequence in case of errors. In addition, simple verbal comments are also used to communicate the start and the end of the demonstration, rehearsal and execution phases of the learning process.

The different parts of the vehicle are initially distributed on a table where teacher and robot work on opposite sides (Fig. 2). The joint execution of the assembly task is possible since the learning process is integrated in the existing DNF architecture for 


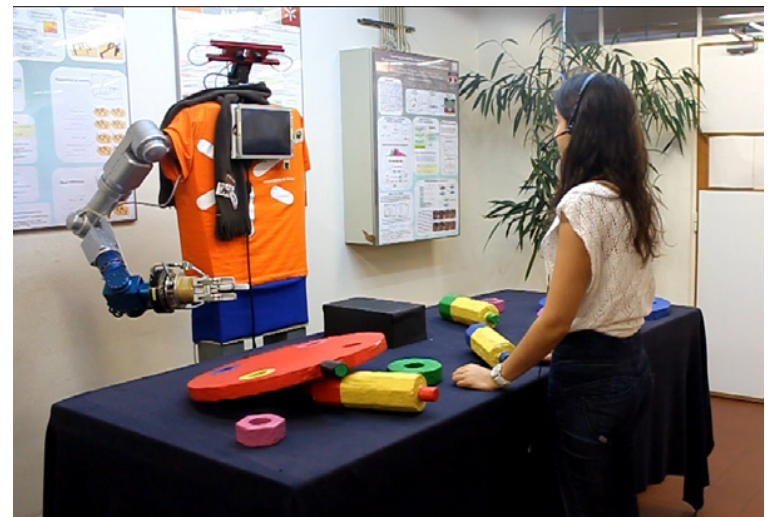

Fig. 2. Experimental setup of the human-robot experiment. ARoS and the teacher are working on opposite sides of the table.

human-robot collaborations. If the robot's prediction about the next assembly step is correct, a decision process is triggered in which the robot selects the best course of action for performing the specific subtask. For instance, the robot will ask the human to handover a part that is not within its reach, or conversely will transfer a part to the co-worker if the assembly step is to be performed in her/his workspace.

The learning process can be divided in three distinct phases which are linked to different computations in the dynamic field model: (i) During the observation phase, the robot stores the serial order of observed events in short-term memory (STM) represented by a stable, multi-bump pattern of neural population activity. (ii) The observation phase is followed by a rehearsal process in which stored patterns from the STM are used as input to simulate the serial order of events in a long-term memory (LTM) layer consisting of two connected fields. Newly established connections between neural populations representing the endeffect of successive assembly steps represent the knowledge about the task structure. (iii) During task execution, negative feedback by the teacher about a predicted subgoal affects the learning process in subsequent demonstration and rehearsal periods. A prediction error results in a longer time window for associative learning defined by the time course of population activity. The prediction may thus become associated with a larger number of already executed steps. This increasing dependence on execution history in multiple demonstration-rehearsal-execution cycles will ultimately eliminate the prediction error.

\subsection{Robotic platform}

For the experiments we used the humanoid robot ARoS (Anthropomorphic Robotic System) built at the University of Minho (Silva, Bicho, \& Erlhagen, 2008). It consists of a static torso on which a 7 DOFs AMTEC arm (Schunk GmbH) with a 3-fingered dexterous hand (Barrett Technology Inc.) is mounted. ARoS is equipped with a stereo camera system fixated on a pan-tilt unit and a speech synthesizer/recognizer (Microsoft Speech API 6.2) for basic forms of verbal communication (Bicho et al., 2010). It is capable of recognizing and producing event and action related words and sentences. Speech input is supplied by a head-set microphone, worn by the human.

The components of the assembly work were designed to limit the workload for the robot's vision system and to enable the robot to perform all assembly steps itself. The vision system provides the $3 \mathrm{D}$-coordinates and the pose of the different components on the table. Objects are recognized using a combination of color and size information. In joint action execution, using this information the robot makes an internal simulation based on its kinematic model that allows it to check off-line if a particular object is reachable and if there is a feasible movement for the intended sub-task execution. This biases the decision of the robot about the best course of action to take (e.g. request the object if not reachable, reach-grasp-place, reach-grasp-handover). Details about the model for decision making in the context of human-robot joint action and the module for movement planning can be found in Bicho et al. (2011) and Silva et al. (2014), respectively. The recognition of the achievement of the assembly steps is based on the positioning of the assembly parts relative to each other (Fig. 6). If, for instance, a wheel is perceived near the base peg, the robot assumes that it is attached to the Base (BA). This is obviously a simplification for the vision system because no explicit recognition of the assembly state has to be performed. However, in cluttered environments this assumption is not completely unrealistic for a cognitive system since occlusions often prevent the vision system from directly observing the end state of a specific assembly step. In this case, the state has to be inferred from other cues (e.g., motion cues, see the Discussion).

For the control of the robotic arm-hand system we applied a movement planning approach in posture space that allows us to integrate cognitive constraints (e.g., the grip type in dependence of the motor intention) and optimization principles derived from human upper-limb movements directed towards objects. The goal is to generate collision-free movements of the robotics arm that are perceived by a human user as smooth and goal-directed (Silva et al., 2014). The robot is able to grasp components that are in its workspace and from the human hand, and has also plugging and handing-over sequences in its motor repertoire.

\section{The Dynamic Neural Field framework}

Dynamic neural fields (DNFs) represent a theoretical framework for developing cognitive control architectures that is consistent with fundamental principles of cortical information processing in distributed networks of connected neuronal populations (Erlhagen \& Bicho, 2006; Schöner, 2008). Task-relevant information is represented by supra-threshold activity patterns (or bumps) of neural populations. These patterns are initially triggered by a transient input from external sources (e.g., vision) or connected neural assemblies but may become self-sustained in the absence of any external input due to strong recurrent interactions within the local populations. The self-stabilizing properties of neural activity allow us to endow autonomous robots with cognitive functionalities that are fundamental for the acquisition of knowledge about temporally extended behaviors such as memory, decision making, and the learning of associations between events separated in time (Curtis \& Lee, 2010; Miller, 2000). Fig. 3 illustrates the temporal evolution of activation in response to two bellshaped inputs that are applied during the time interval $\left[t_{0}, t_{e}\right]$ to different field locations. The labels $x_{S G 1}$ and $x_{S G 2}$ shall indicate that the local populations representing the assembly steps or subgoals SG1 and SG2, respectively, become activated by the two external inputs. In the left panel one can see that two bumps stabilize after the cessation of the inputs at time $t_{e}$. This bimodal pattern represent the short-term memory of the two external events. To implement such an activation-based from of memory, the field dynamics must be in a bistable regime (Amari, 1977), that is, the bump state must co-exist with a homogeneous resting or baseline state so that sufficiently strong excitatory or inhibitory input may switch between the two states. The right panel illustrates the situation of decision making when one subgoal among multiple alternatives has to be selected. In this example, the strong competition between distant field locations mediated by lateral inhibition ensures that only the population representing SG2 reaches a stable activation bump. Note that at time $t=0$, when the two external inputs of 


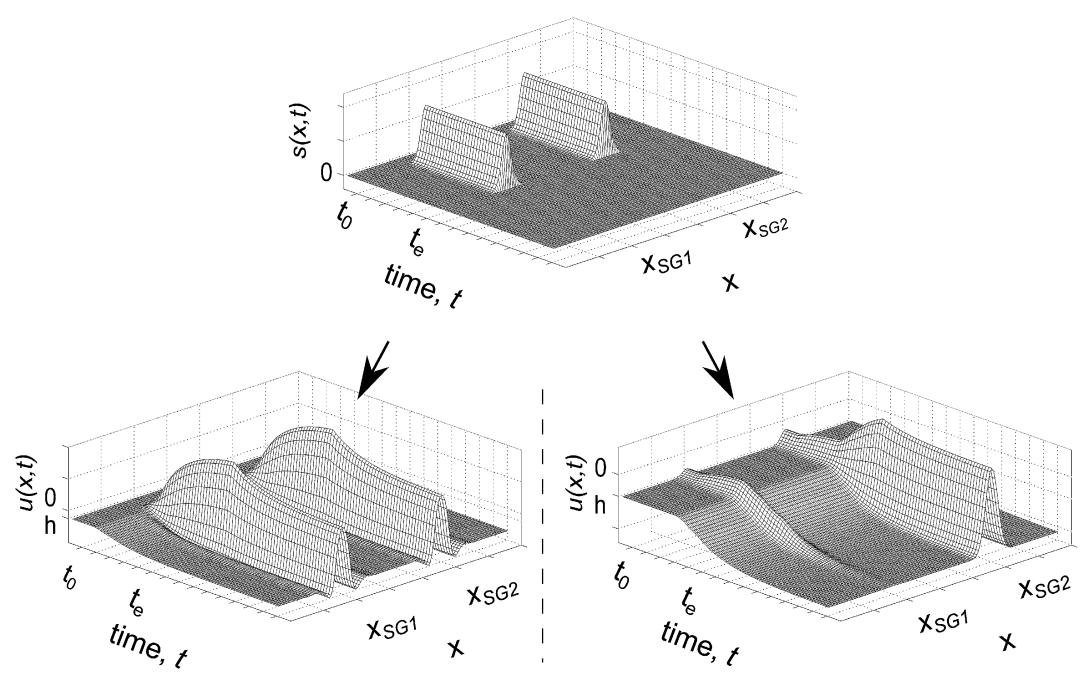

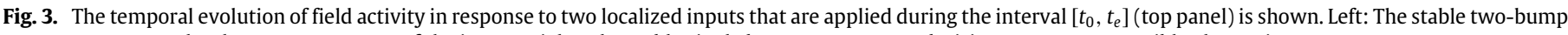
pattern represents the short-term memory of the inputs. Right: The stable single bump represents a decision among two possible alternatives.

equal strength are applied, the two populations appear to be already preshaped by weaker localized inputs from other sources. Weaker input does not drive populations beyond the threshold for persistent activity but may nevertheless bias the decision process (Erlhagen \& Schöner, 2002). In the example, the preshaping is relatively stronger for subgoal SG2. To illustrate and explain the dynamic behavior of the learning model in the following sections, we will not show the complete 3D plots of the temporal evolution of activity but either a snapshot of the activation pattern at a particular time or the complete time course at a specific field location.

A context dependent variation of the resting state is applied in the learning model to express different levels of "readiness" of the cognitive system in the distinct phases of the learning experiment. This is in line with computational and neurophysiological studies that discuss this mechanism as a neural correlate for cognitive control (Salinas, 2003; Wardak, Ramanoël, Guipponi, Boulinguez, \& Ben Hamed, 2012). A higher baseline activity brings neural populations closer to the threshold. This accelerates in general the time course of the population response to input and makes it possible that also relatively weaker input may trigger the evolution of a bump.

The learning model consists of three interconnected dynamic fields of a particular type first proposed and analyzed by Amari (Amari, 1977). In each field $i$, the temporal evolution of activity, $u^{i}(x, t)$, of a neuron at field position $x$ is governed by the following differential equation:

$$
\begin{aligned}
\tau^{i} \frac{\partial u^{i}(x, t)}{\partial t}= & -u^{i}(x, t)+\int w^{i}\left(x-x^{\prime}\right) f_{0}\left[u^{i}\left(x^{\prime}, t\right)\right] d x^{\prime} \\
& +h^{i}(x, t)+s^{i}(x, t)+\zeta^{\text {stoch }}(x, t)
\end{aligned}
$$

where $\tau^{i}>0$ is the time constant of the dynamics and $h^{i}(x, t)<0$ defines the baseline level to which field activity relaxes without the summed input $s^{i}(x, t)$ from external sources and connected populations. $\zeta^{\text {stoch }}(x, t)$ represents stochastic noise to force a decision whenever the representations of several possible assembly steps compete. The integral term describes the recurrent interactions within a local neural population to which only sufficiently active neurons contribute. The non-linear transfer function $f_{0}$ is chosen as the Heaviside step function $f_{\theta}$ with threshold $\theta=0$ defined by:

$f_{\theta}[u]= \begin{cases}0, & u-\theta \leq 0 \\ 1, & u-\theta>0 .\end{cases}$

The coupling strength between any two neurons $x$ and $x^{\prime}$ depends on the distance only, that is, $w^{i}\left(x \mid x^{\prime}\right)=w^{i}\left(x-x^{\prime}\right)$. The coupling
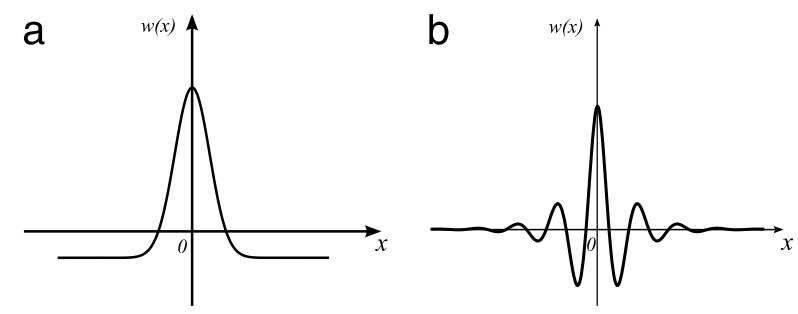

Fig. 4. Interaction kernels $w(x)$. (a) Gaussian coupling function with lateral inhibition. (b) Coupling function with several positive zeros adapted from Laing et al. (2002).

function $w(x)$ can have two different shapes (Fig. 4). A Gaussian type with bell-shaped excitation and constant lateral inhibition (Fig. 4a) ensures that only a single stable bump may exist at all time. It is used in the model layer that implements a decision process. It is given by

$w(x)=A e^{\frac{-x^{2}}{2 \sigma^{2}}}-w_{\text {inhib }}$

where $w_{\text {inhib }}>0$ is a constant inhibition, and $A>0$ and $\sigma>0$ describe the amplitude and standard deviation of the Gaussian, respectively. The second type with several positive zeros introduced by Laing and colleagues (Laing, Troy, Gutkin, \& Ermentrout, 2002) (Fig. 4b) allows the existence and stability of multiple bumps of persistent population activity. It is applied in the fields with a multi-item memory functionality. It is given by

$w(x)=A e^{-b|x|}(b \sin (|\alpha x|)+\cos (\alpha x))$

where $A>0$ defines the amplitude, and $b>0$ and $0<\alpha<=1$ define the rate at which the oscillations decay with distance and the zero crossings of $w(x)$, respectively. Note that since in the present application only a relatively small number of items have to be stored simultaneously, a coupling function of lateral inhibition type with a limited spatial range could have been chosen as well. We use the oscillatory coupling functions to be consistent with previous work in which we exploited the existence of an upper bound for the working memory capacity when all bumps "see" each other (Ferreira, Erlhagen, Sousa, \& Bicho, 2014).

\section{DNF model of task learning}

Fig. 5 presents a schematic view of the model architecture with two interconnected modules. It reflects the idea of two 
complementary learning systems that has been proposed as a solution for natural and artificial learning systems to avoid the potentially "catastrophic" interference of old and new memories (McClelland et al., 1995). Converging lines of neurophysiological evidence suggest that Prefrontal Cortex-Hippocampus interactions might constitute a neural substrate for this hypothesis. Hippocampus is viewed as forming specific memories of sequential events and areas in Prefrontal Cortex accumulate the shared structure of related memories from a set of connected experiences (for a recent review see (Preston \& Eichenbaum, 2013)).

Consistent with this view, the Short Term Memory (STM) module, formalized by a single dynamic field $u^{s t m}$, is responsible for memorizing observed sequences of executed subtasks in a stable multi-bump pattern. The Long Term Memory (LTM) module formed by two coupled dynamic fields, $u^{p a}$ and $u^{p r}$, encodes serial order knowledge from multiple demonstrations in newly established connections between neural populations in the two layers. $u^{p a}$ encodes already achieved (or past) sub-goals whereas $u^{p r}$ present sub-goals that still have to be accomplished. During demonstration, the pattern formation process in all fields is initiated by the input from the vision system whereas during off-line learning periods, the stored multi-bump pattern in STM acts as input to $u^{p r}$ and thus drives the population dynamics in LTM.

Interestingly, the basic processing mechanisms used in the two modules combine the specific strengths of two model classes that have been widely used in the past to explain human serial order memory and performance. The storage and recall of sequences as parallel activation patterns of all elements is the hallmark of so-called competitive cuing models (Grossberg, 1978, for review Rhodes, Bullock, Verwey, Averbeck, \& Page, 2004). Activationbased memory favors fast, "one-shot" learning from demonstration. Encoding sequential information in synaptic connections and a fundamentally serial recall mechanism is the hallmark of recurrent neural network models (Cleeremans \& McClelland, 1991; Elman, 1990). Weight-based learning usually requires many repetitions of the same sequence to be stable. However, as a slow process it is also able to smoothly integrate new knowledge about successive events into an existing representation.

The vision system provides information about the current state of the assembly work to all field layers. A perceived change in the state of the task, that is, the achievement of a certain subtask, is modeled as a Gaussian input $s^{v i}(x, t)$ to the respective neural populations (Fig. 6).

In the following, we describe the functioning of the two modules and the implemented learning mechanisms in more detail. The mathematical description of field model as well as the values for the model parameters can be found in the Appendices.

\subsection{Short-Term Memory (STM) module: fast learning of serial order}

The function of STM in the robotics experiments is to first store the serial order of a single demonstration and then to provide the memorized information as input to LTM during an off-line rehearsal period. For the recurrent interactions of field $u^{\text {stm }}$ we used the connection function with several zero-crossings (Eq. (4)) described in Section 3 to guarantee that all sequentially presented perceptual events can be stored in parallel in a multi-bump pattern. To encode serial order in this pattern, we adopt a threshold accommodation dynamics tested in previous work in which the robot had to memorize the order and relative timing of a musical sequence (Ferreira et al., 2014). When the activity of a certain population reaches threshold, the $h$-level of suprathreshold neurons is adapted using a simple linear dynamics (for mathematical details see Appendix A). The net effect is a continuous increase of the bump representation as a function of elapsed time since event onset. In the robotics experiments, this

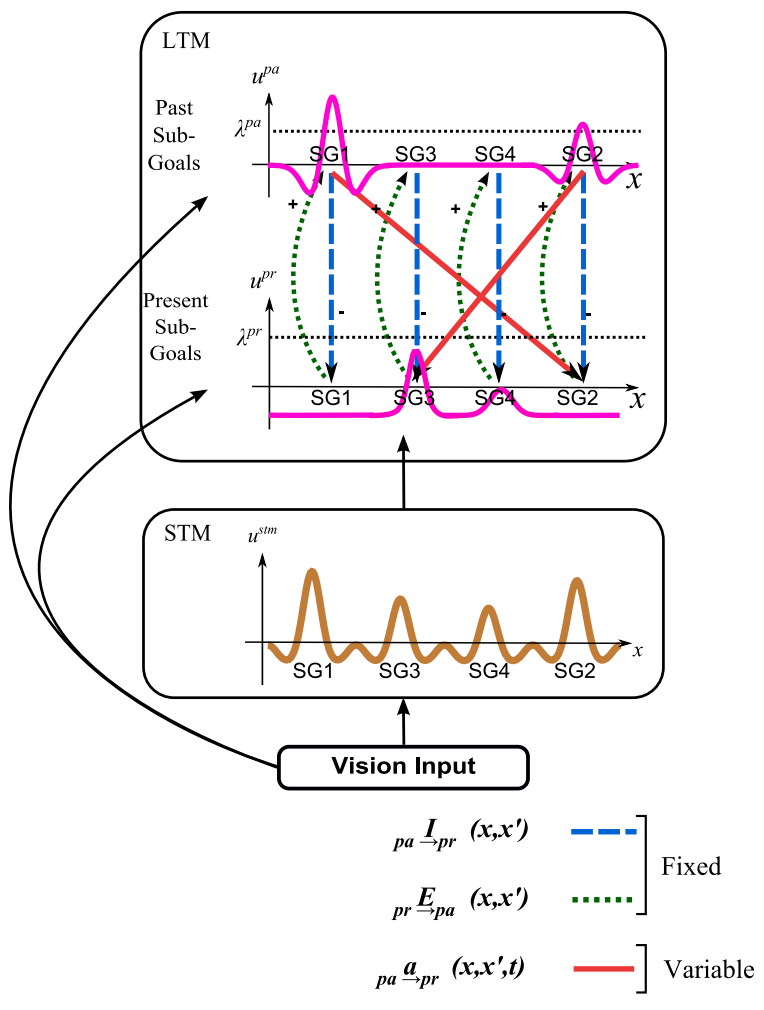

Fig. 5. Schematic view of the sequence learning model. It consists of two interacting modules, the Short Term Memory (STM) and the Long Term Memory (LTM). Details of their functioning are explained in Sections 4.1 and 4.2, respectively.

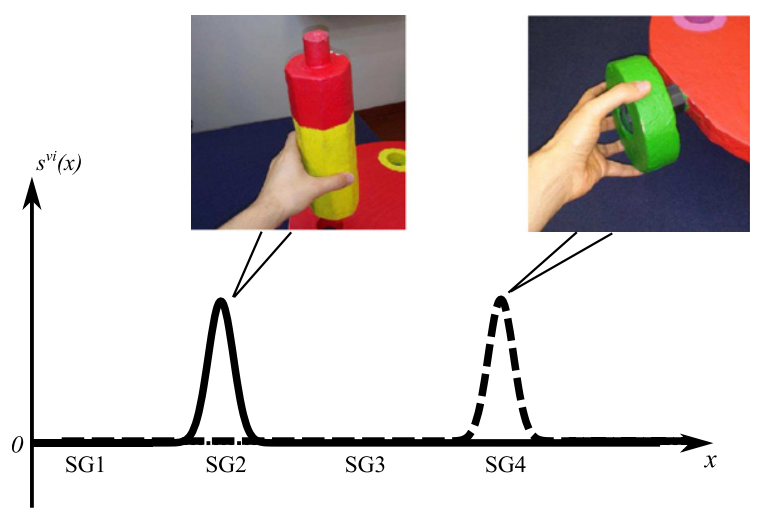

Fig. 6. Example of input from the vision system to the fields. The completion of subtasks SG2 and SG4 is modeled as Gaussian inputs to the respective neural populations representing these assembly steps.

increase is stopped when the demonstrator communicates the end of the demonstration. The relative strength of individual bumps in the stable multi-bump pattern thus represents the temporal order (and relative timing) of events (Fig. 7). When this pattern is applied as input to $u^{p r}$, a scaling factor proportional to the sequence duration is used to guarantee that all individual input bumps are above threshold, that is, all sequence elements are activated in parallel. From an application point of view, it is important to stress that the gradient can be established also iteratively from more then one demonstration to correct for instance potential failures of the vision system (Ferreira et al., 2014).

\subsection{Long Term Memory (LTM) module: dynamics of long-term memory formation}

In the robotics experiments, one or several users may demonstrate different orders of task execution. Any useful model 


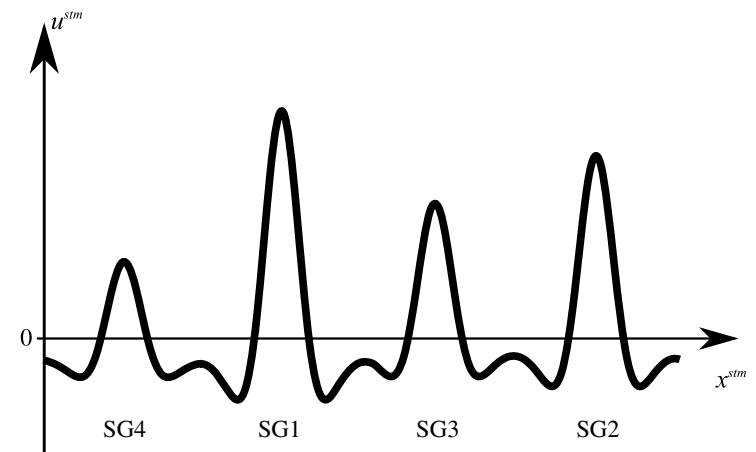

Fig. 7. Snapshot of a stable multi-bump pattern in STM. In response to a sequence of visual inputs, the field dynamics establishes an activation gradient which represents the demonstrated serial order of task execution (SG1 to SG4).

of memory thus needs to account for sequential learning, where new information can be integrated with old information at any time. In the LTM module, strengthening and weakening of synaptic weights between population representations of subtasks in off-line rehearsal periods implements this functionality. We take inspiration from recent neurobiological findings suggesting that during behavioral planning previous and future goals or end states of intended actions are represented by separate neural populations in prefrontal areas (Genovesio, Brasted, \& Wise, 2006). The DNF model reflects these findings by assuming the existence of two fields, $u^{p a}$ and $u^{p r}$, with neural populations encoding already achieved and still to be accomplished subtasks, respectively (for mathematical details see Appendix B). The working memory functionality of $u^{p a}$ requires a connection function with multiple zero-crossings (Eq. (4)) whereas the selection of the next subtask among all remaining ones requires an intra-field connectivity in $u^{p r}$ of lateral-inhibition type (Eq. (3)). A Hebbian learning mechanism following the principle of the Delta rule (Widrow \& Hoff, 1960) is applied to establish and re-distribute synaptic connections between neural populations in $u^{p a}$ and $u^{p r}$ (solid red lines in Fig. 5). To gate the associative learning, we exploit the notion of a learning threshold (Seitz \& Dinse, 2007). The population of an already simulated subtask becomes associated with a population response of a newly planned one as long as the two population activities do not fall below a certain learning threshold. The exact time window for learning is determined by the dynamics of corresponding neural populations in $u^{p a}$ and $u^{p r}$. The two populations are coupled by fixed excitatory and inhibitory connections (dashed green and blue lines in Fig. 5). During off-line rehearsal, the stored multi-bump pattern in STM is used as stationary input to field $u^{p r}$. Since the strong lateral inhibition in this field only supports single bump solutions, the first bump evolves at the position with the strongest input (SG1 in Fig. 7). Fig. 8 compares the time course of the maximal level of activation of populations in $u^{p a}$ and $u^{p r}$ representing the first subtask. At time $t_{0}$, the population in $u^{p r}$ receives input from $u^{\text {stm }}$ and its activity begins to rise. It drives through the excitatory connections $\underset{p r \rightarrow p a}{E}$ the population in $u^{p a}$. The activity in $u^{p a}$ continues to grow due to the excitation from $u^{p r}$ and the recurrent interactions within the population. The strong inhibitory feedback connections $\underset{I}{I}$ cause in turn a decrease of the activity $u^{p r}$ to resting level, resulting in a transient activity pattern in layer $u^{p r}$. Also the activity in $u^{p a}$ decreases to some extent due to a local $h^{p a}$-adaptation dynamics of the neurons forming the localized pattern. However, the recurrent interactions are sufficiently strong to stabilize the memory bump with a strength below the learning threshold $\lambda^{p a}$. Since the population representing SG1 now receives inhibitory

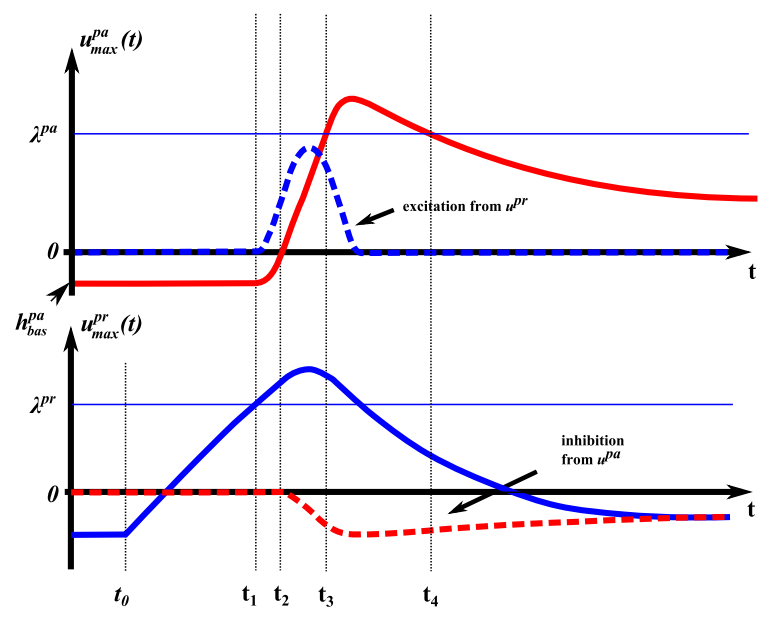

Fig. 8. Interaction process between corresponding populations in $u^{p a}$ and $u^{p r}$ during off-line learning. The maximal level of activation of the two populations representing the same subgoal is plotted as a function of time (solid lines). The dashed line in the top panel represents the time course of excitatory input that the population in $u^{p a}$ receives from the corresponding population in $u^{p r}$ whereas the dashed line in the bottom panel represents the time course of the inhibitory feedback. $\lambda^{p a}$ and $\lambda^{p r}$ represent thresholds for the associative learning of new connections to different subgoal representations in $u^{p r}$ (for more details see the text).

input from its counterpart in $u^{p a}$, the second strongest input from STM will drive the population representation of SG2 to a suprathreshold activity level which then becomes suppressed due to the feedback inhibition. During this off-line process, eventually all subtasks stored in the multi-bump pattern of STM become sequentially activated and are memorized in $u^{p a}$. Associative learning between an active population in $u^{p a}$ and newly evolving population representations in $u^{p r}$ takes place whenever their activities are above the respective learning threshold, $\lambda^{p a}$ and $\lambda^{p r}$. For the simulation example in Fig. 8, the time window for establishing associative links with SG1 is $\left[t_{3}, t_{4}\right]$.

Following the off-line learning period, the robot tries to execute together with a co-worker the sequential task. The robot thus has to switch from a passive rehearsal mode to an active role in which it generates overt behavior guided by the task memory. To realize this switch, we take inspiration from computational and neurophysiological studies suggesting that the resting state of neural populations may be controlled by the nervous system in a highly task dependent manner (Salinas, 2003; Wardak et al., 2012). A higher resting state of $u^{p a}$ brings populations closer to the activation threshold and thus accelerates the formation of a bump in response to visual input. As a consequence, also the inhibition to the corresponding population in $u^{p r}$ starts earlier and the evolution of the bump appears to be suppressed (Fig. 9). However, through the newly established excitatory connections to populations in $u^{p r}$, the bump will drive the population of the next subtask. In the robot experiments, this process is interpreted as a prediction of the cognitive system based on execution history which of course can be correct or wrong.

\subsubsection{Learning rule}

To implement a sequential acquisition of knowledge about possible serial orders and the longer term dependencies between subtasks, the synaptic learning process in LTM should not only promote the formation and strengthening of new associations between successive events but also the adequate adjustment of previously established connection weights. The goal is that in successive learning cycles, the input necessary to drive a particular 


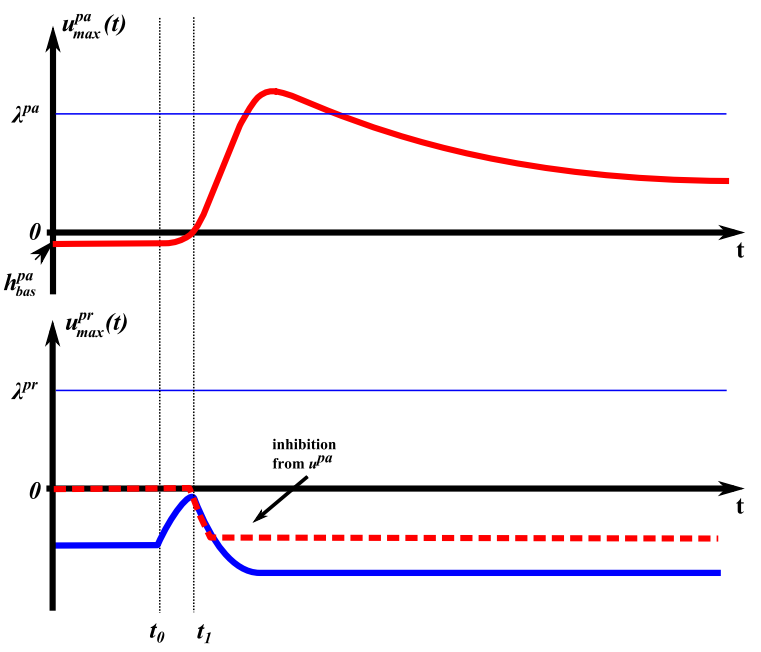

Fig. 9. Interaction process between corresponding populations in $u^{p a}$ and $u^{p r}$ during task execution. Note that the baseline activity of $u^{p a}$ appears to be increased compared to the resting state during rehearsal (compare Fig. 8).

population in $u_{p r}$ to a suprathreshold activation level is redistributed among several active populations in $u_{p a}$.

The mathematical formulation of the learning rule that we use to establish synaptic connections $\underset{p a \rightarrow p r}{a}$ between any two sufficiently active neurons in the past and present layer is given by:

$$
\begin{aligned}
\tau_{a} \frac{\partial \underset{p a \rightarrow p r}{a}\left(x^{\prime}, x, t\right)}{\partial t}= & f_{\lambda p r}\left[u^{p r}(x, t)\right] f_{\lambda} p a\left[u^{p a}\left(x^{\prime}, t\right)\right] \\
& \times\left[e^{p r}(x, t)-\eta_{p a \rightarrow p r}^{a}\left(x^{\prime}, x, t\right)\right] .
\end{aligned}
$$

The first term on the right of (5), represents the Hebbian learning mechanisms with thresholds, since synaptic connections are updated only when $u^{p r}>\lambda^{p r}$ and $u^{p a}>\lambda^{p a}$ are satisfied. The error function $e^{p r}$ defines the value of the update according to the principle of the Delta rule:

$e^{p r}(x, t)=g_{\lambda} p r\left[u^{p r}(x, t)\right]-\int f_{\lambda p a}\left[u^{p a}\left(x^{\prime}, t\right)\right] \underset{p a \rightarrow p r}{a}\left(x^{\prime}, x, t\right) d x^{\prime}$

where the target value is defined by a S-shaped target function

$g_{\lambda p r}[u]=\frac{\left[u-\lambda^{p r}\right]^{+}}{1+\left[u-\lambda^{p r}\right]^{+}}$

which ensures that the weight growth does not depend on the amplitude of $u^{p r}$. Although the Heaviside function could have been used as well, (7) produces a smoother bell-shaped target function. The weights $\underset{p a \rightarrow p r}{a}$ will thus be updated to produce excitation with a similar smooth profile.

\subsubsection{Integrating feedback from the teacher}

What happens when during recall a prediction error occurs, for instance, because the newly learned associations do not represent well the longer dependencies between subtasks? Think for example of two initial demonstrations in which the last column before placing the top platform (TP) differs. During recall, the placing of either of the two columns will lead to the prediction of TP as the next subgoal which of course represents an omission error since the other column is still missing. The solution is to increase the time window for associative learning to allow that longer-term relationships between subtasks may be established during the offline learning period. In the experiments, the robot gets immediate verbal feedback from the tutor about its prediction. It is known that the time course of persistent activity can be modulated by previous action outcomes across multiple trials (Curtis \& Lee, 2010). We assume here that negative feedback effectively increases the time that persistent activity remains above the learning threshold which could be achieved by adapting different parameters of the field dynamics (see the Discussion). For simplicity, we directly change the time scale $\tau_{h p a}$ of the $h^{p a}$-dynamics which controls the decay rate of the population response. Initially, the value is relatively small, so that only associations between directly succeeding subtasks are learned. With larger $\tau_{h}$ pa values, the time window for learning becomes longer, and consequently associations to several subtask representations in $u^{p a}$ will be established (compare Fig. 10b and d). The learning rule ensures that the total association strength necessary to trigger a prediction is now divided among several populations. The left column of Fig. 10 illustrates the mechanism for learning pairwise associations for the example of subtasks SG3 and SG4. Note that the activity of SG2 is already below the learning threshold at the time when the representation of SG4 in $u^{p r}$ becomes suprathreshold (vertical line in the bottom panel). Following the negative feedback by the tutor, the $\tau_{h p a}$ parameter is adapted leading to a slower decay of population activity during a new demonstration and the subsequent off-line rehearsal phase. As depicted in the right column of Fig. 10 for the example of subtasks SG2, SG3 and SG4, the Hebbian learning now supports associations to two previous elements of the sequence. For the present example, however, this is still not sufficient to avoid prediction errors. Ultimately, this feedback driven adaption-learning cycle will lead to the prediction that all four columns have to be in place before TP can be placed.

\section{Results}

We report results of three learning experiments in which the robot ARoS observed different tutors executing the assembly sequence with varying temporal orders. In the last experiment, an error occurred and was eventually corrected following additional demonstrations. Each experiment consists of observation, off-line rehearsal and execution phases. During observation, the human has all parts within reach to perform the whole sequence. During execution, objects are distributed on the table, and human and robot cooperate with each other to complete the task. A collaborative task execution was chosen to show that the task knowledge acquired in a social learning situation may be used by the robot to coordinate fluent joint action with a human co-worker (Bicho et al., 2011). Human feedback is given verbally through a set of predefined sentences (e.g. "that is correct/wrong") recognized by the speech recognition system. When the robot vocalizes a prediction (e.g. "next we can place the blue column") it always waits for a reply before performing the action. If the prediction is wrong, the negative feedback from the tutor changes the decay rate of population activity in the past layer of LTM in subsequent learning sessions. The robot requires a new demonstration by verbalizing the sentence "Can you show me again?" and the tutor starts a new demonstration. Switching between the demonstration, simulation and execution phases of the experiments is also controlled by simple verbal communication. The tutor communicates the beginning of a demonstration with the phrase "I will show you" and ends it with "I have finished", which triggers the off-line simulation of the just demonstrated sequence. Following a fixed number of covert rehearsals, the robot warns the tutor by vocalizing "I think I am ready", which starts the joint execution of the assembly task.

For the presentation of results we focus on the construction of the top part of the toy vehicle. A video of the complete task can be found in the supplementary material, see Appendix D. 

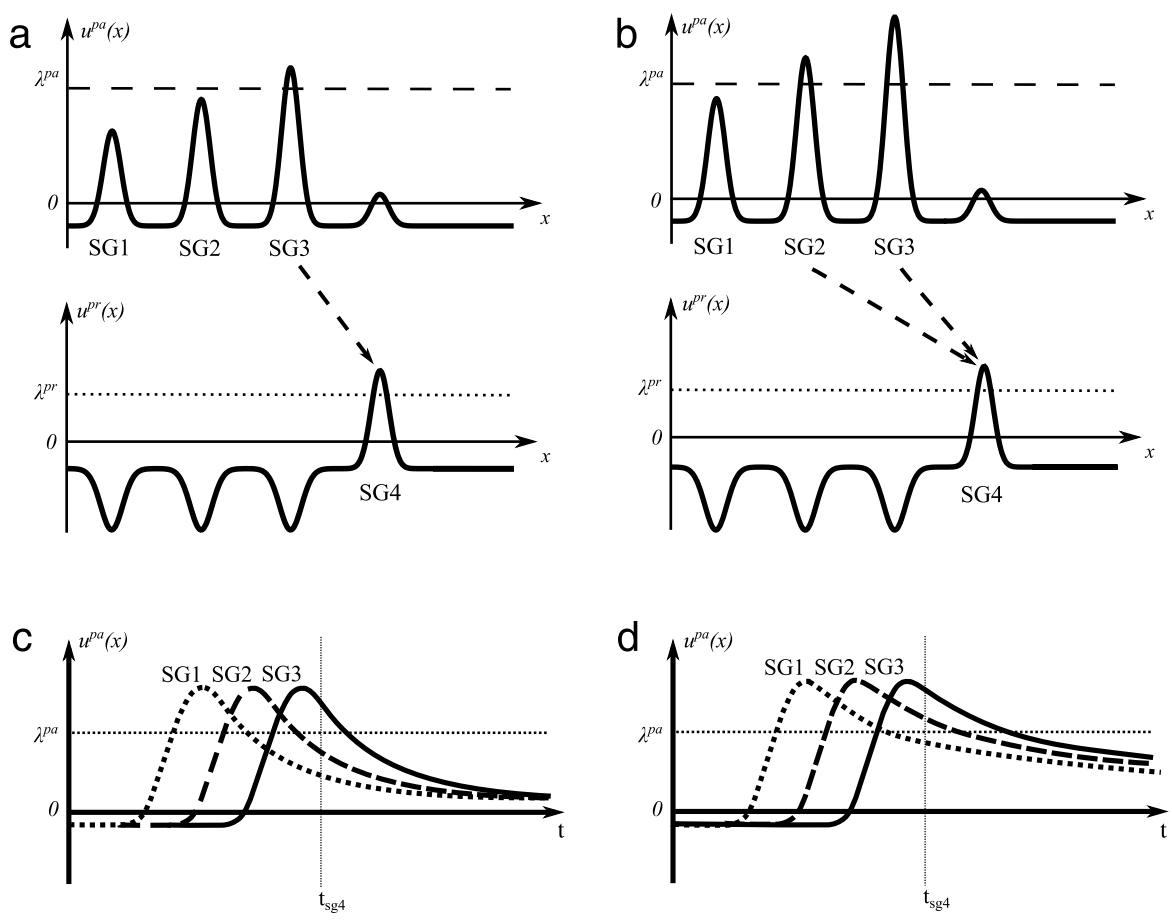

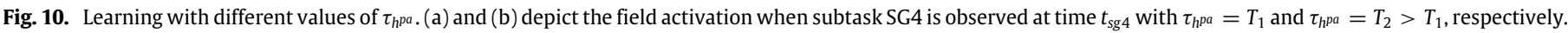

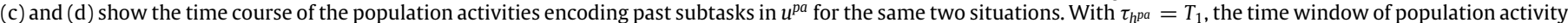

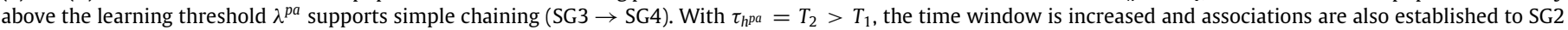
(compound chaining).

\subsection{First experiment: learning a specific order}

In the first experiment, the tutor demonstrates the assembly of the top part (Fig. 11), using the following serial order of subtasks (see video 1, Appendix D):

$\mathrm{BA} \rightarrow \mathrm{MC} \rightarrow \mathrm{GC} \rightarrow \mathrm{RC} \rightarrow \mathrm{BC} \rightarrow \mathrm{TP}$

The demonstration took approximately 28 seconds. Fig. 12 depicts the resulting memory pattern in STM which correctly represents the observed serial order in the relative activation strengths.

Next during off-line rehearsal, the robot performed 30 simulation trials in which the stable multi-bump pattern in $u^{\text {stm }}$ is used as stationary input to $u^{p r}$. Since the strong lateral inhibition in this field supports only single bump solutions, the first bump evolves at the position with the strongest input representing the first element of the sequence. Due to the excitatory-inhibitory couplings between corresponding populations in the two LTM layers, the representation of the first subtask becomes eventually suppressed and the second strongest input representing the second sequence element may win the competition. Each rehearsal trial has a fixed duration $T^{\text {reah }}$ during which all subtask representations in $u^{p r}$ become sequentially activated (see Section 5.4). After $T^{\text {reah }}$, all remaining suprathreshold activations in $u^{p a}$ and $u^{p r}$ are set back to resting level. Fig. 13 compares the temporal evolution of the maximally excited neuron of each population in $u^{p a}$ and $u^{p r}$, during observation (Fig. 13a) and rehearsal (Fig. 13b). As can be clearly seen, the serial order is preserved during the sequence activation from memory. The time courses, however, appear to be accelerated by a factor 5 since during rehearsal the fields evolve autonomously with the proper time scale. In contrast, during observation the evolution of a bump in $u^{p r}$ is triggered by visual input about the achievement of a certain subgoal which of course depends on how long the tutor took to perform the subtask.
To start the joint task execution, the tutor placed the base BA (Fig. 14a), leading to the emergence of a bump at the location of the BA population in $u^{p a}$ (see top panel in Fig. 15). This activation pattern drives through the newly established excitatory connections $\underset{p a \rightarrow p r}{a}$ the evolution of a bump at the location of the MC population in $u^{p r}$ (see bottom panel in Fig. 15), corresponding to a prediction which is in line with the demonstrated order.

The tutor responds to the vocalized prediction (Fig. 14a) with positive feedback (see Video 1, Appendix D). Since the magenta column was in the robot's workspace but had to be inserted on the tutor's side, the robot transferred the MC to the co-worker who inserted it in the corresponding hole of the base (Fig. 14b). The working memory of the executed assembly step in $u^{p a}$ drove a new prediction represented by the GC population in $u^{p r}$ (Fig. 16). Following the positive feedback by the tutor (Fig. 14c), since the green column was located outside its reaching area $\mathrm{AROS}$ requested the GC by raising its empty hand towards the co-worker and verbalizing the request (see (Bicho et al., 2011) for details about the integration of verbal and nonverbal communication in the DNF architecture for HRI). ARoS received the column (Fig. 14d) and attached it to the base (Fig. 14e). The human-robot collaboration followed the initially demonstrated order until all subgoals were successfully performed.

\subsection{Second experiment: learning an alternative order}

In the second experiment, another tutor demonstrated the same task, but using a different order (see video 2, Appendix D). Compared to the first sequence, the order of manipulation of the two columns $\mathrm{RC}$ and $\mathrm{BC}$ was reversed:

$\mathrm{BA} \rightarrow \mathrm{MC} \rightarrow \mathrm{GC} \rightarrow \mathrm{BC} \rightarrow \mathrm{RC} \rightarrow \mathrm{TP}$ 
a

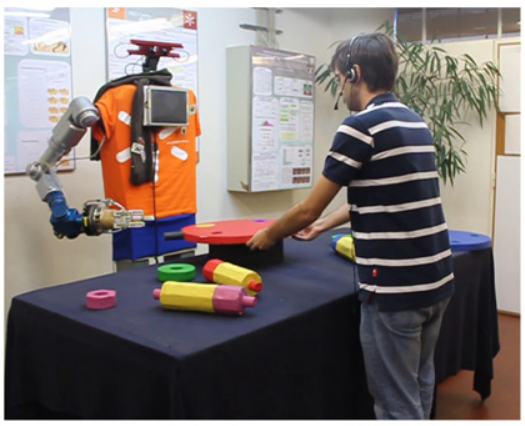

C

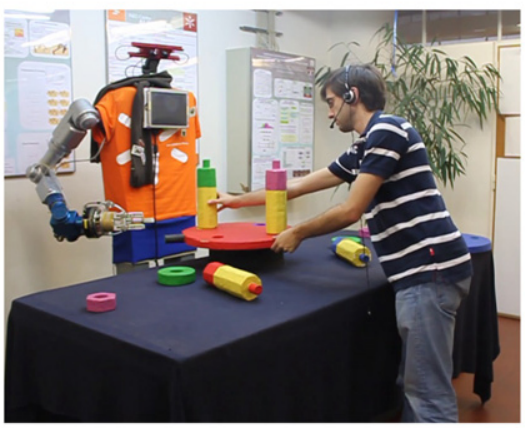

e

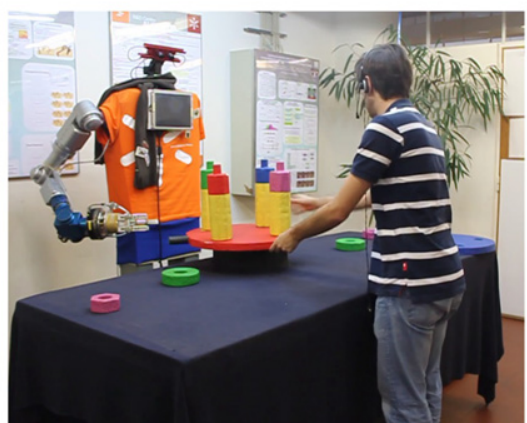

b

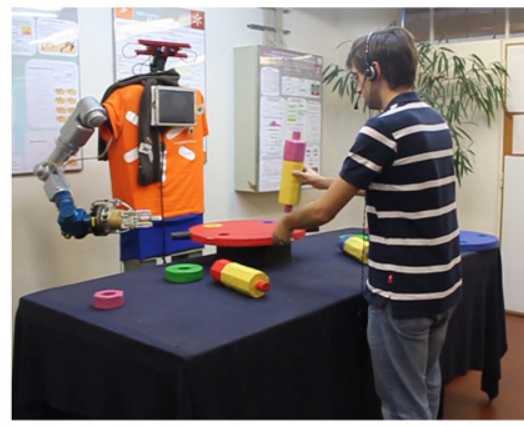

d

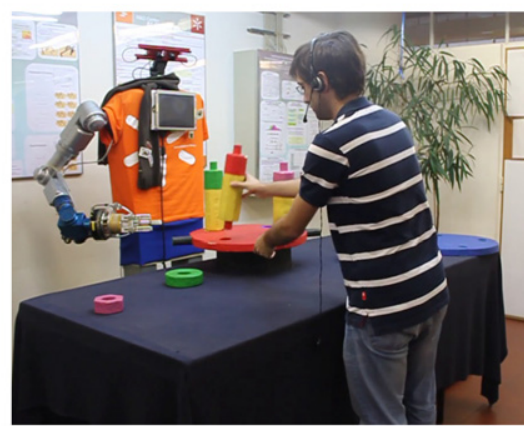

f

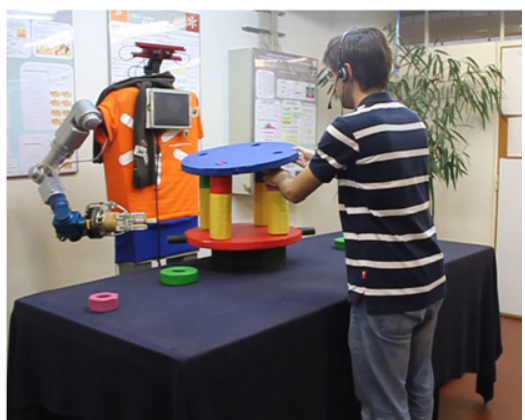

Fig. 11. Snapshots of the first demonstration.

The second demonstration took again about $30 \mathrm{~s}$. After the initial sequence encoding in STM, the off-line learning incorporates this new information in the existing sequence representation. As a result, placing GC now triggers supra-threshold activity in two populations, RC and BC, meaning that both subtasks are now represented by the learned inter-field connections as possible goals (Fig. 17). The additive noise in equation (1) governing the dynamics of $u^{p r}$ led to a decision to predict BC as the next subgoal. Since BC was in the co-workers's workspace, she grasped and inserted the column (Fig. 18). After BC was placed, RC was chosen as the next subgoal.

To directly test the probability of choosing each of the two possible subgoals, BC and RC, the weights $\underset{p a \rightarrow p r}{a}$ were stored and an off-line test was run a posteriori. The simulated situation was the same as during the real experiment, with BA, MC and GC already in place and the corresponding populations in $u^{p a}$ activated above threshold. The decision process in $u^{p r}$ was repeated 1000 times. At the beginning of each trial, the field was reset to resting level and the summed input from active populations in $u^{p a}$ triggered the evolution of a suprathreshold activation pattern at one of the locations in $u^{p r}$. The percentage of choices for BC and RC was $47.6 \%$ and $52.4 \%$, respectively. This probability of choice is reflected in the balanced connections strengths that link the populations BA, GC and MC in $u^{p a}$ to RC and BC in $u^{p r}$, depicted in Fig. 19.

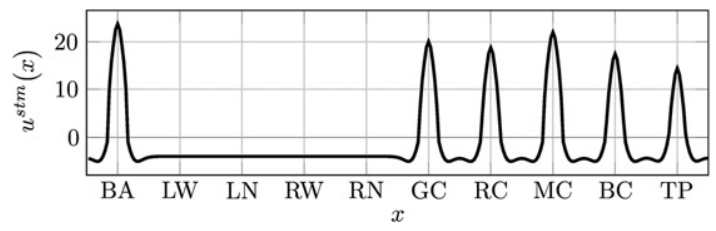

Fig. 12. Stable activation pattern in $u^{\text {stm }}$ after the first demonstration.

\subsection{Third experiment: error occurrence and correction}

A third experiment was conducted with the objective of testing the adaptation mechanisms for correcting errors in the serial task execution (see video 3, Appendix D). To this end, the task was redemonstrated by a third tutor with the following sequence:

$\mathrm{BA} \rightarrow \mathrm{BC} \rightarrow \mathrm{RC} \rightarrow \mathrm{GC} \rightarrow \mathrm{MC} \rightarrow \mathrm{TP}$.

This new demonstration took approximately 34 s. Following the demonstration and the off-line learning period, the tutor placed the base and the robot predicted MC as the next step. After inserting MC, the input from $u^{p a}$ supports GC, BC and TP as possible next subgoals. A bump is eventually formed at TP which obviously represents a premature prediction at the present state of the assembly work (see Figs. 20 and 21a). 

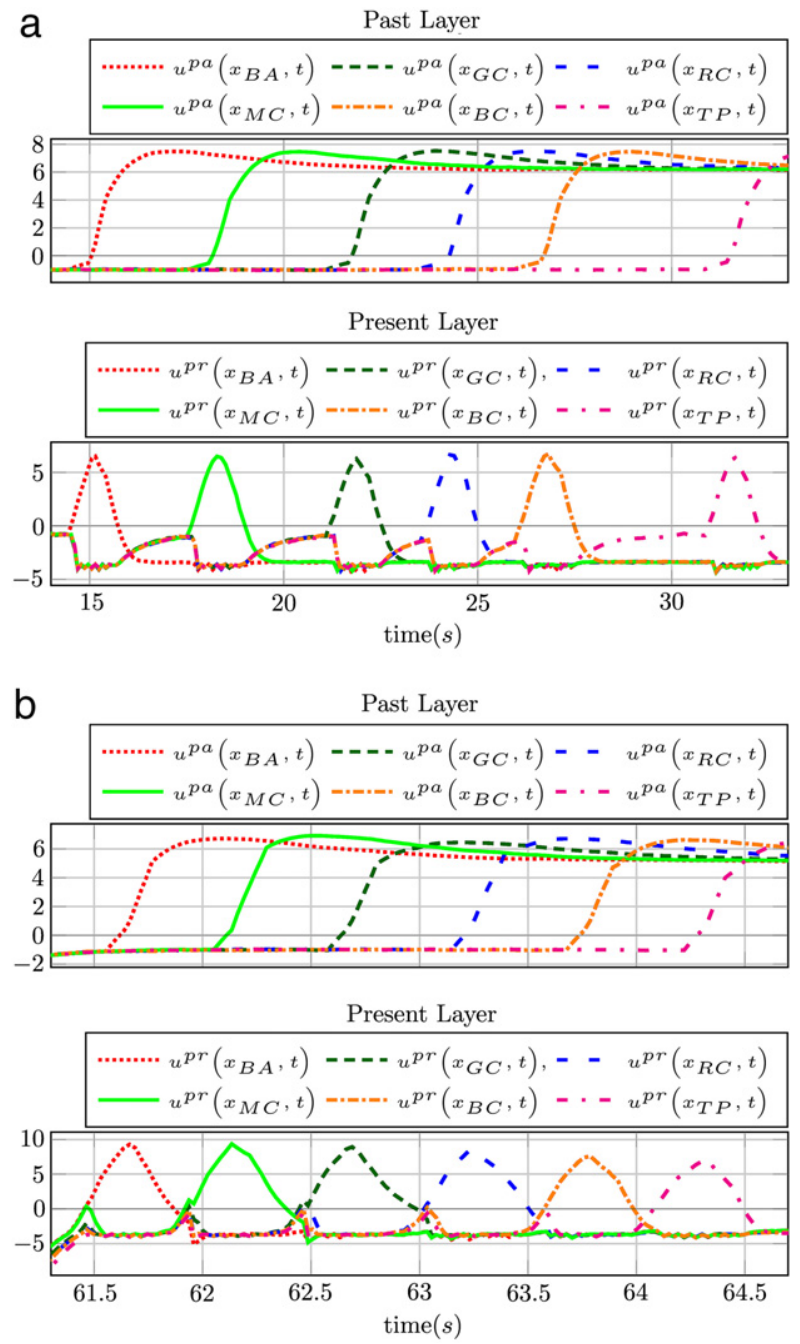

Fig. 13. First learning experiment: Time courses of population activity in the two LTM layers during (a) the observation phase, and (b) during the internal rehearsal phase.

Looking at the individual connection strengths $\underset{p a \rightarrow p r}{a}$ (Fig. 22) it becomes clear that the choice of TP at this stage was in fact the less likely one. However, even if GC or BC would have been chosen, the probability of an omission error in subsequent stages remained high. This was verified through an a posteriori statistical test like described in the second experiment. The values of the weights $\underset{p a \rightarrow p r}{a}$ were stored and the decision process was simulated for the multiple choices at different states of the assembly work. For each possible state, the decision process in $u^{p r}$ was repeated 1000 times to measure the probability of each possible outcome. This allowed us to build the probability tree depicted in Fig. 23. It shows that the total probability of an error occurrence was $89.6 \%$.

Error correction through adaptation of the decay rate of the $h^{p a}$ dynamics. Negative feedback from the tutor increases the time window for associating a specific prediction with a longer history of executed subtasks. This is achieved by adapting the decay rate of the $h^{p a}$-dynamics in subsequent demonstration-rehearsal cycles.

After a new demonstration of the same serial order, the learned connection weights led to a decision to insert BC following MC. However, TP was again prematurely chosen as the next sub-goal (Figs. 21b and 24). This second error occurred because the time window for learning still did not include a sufficient number of subgoals to disambiguate the situation. Fig. 25 depicts the probability tree of the decision process for this second recall attempt, again calculated from a posteriori simulation (Fig. 23). The total probability of error occurrence dropped but remained quite high (66.7\%).

Negative feedback by the tutor caused a further decrease in the decay rate of $h^{p a}$. Following a third demonstration, the adapted and newly established connection weights correctly represent the long-term causal dependency of subtasks (Figs. 21c and 26). Only after all columns were in place, ARoS selected TP to finish the task.

Like in the previous recall attempts, the weights were stored and an a posteriori test was made with each decision condition simulated 1000 times. The resulting probability tree (Fig. 27) shows that the error probability dropped from $89.6 \%$ and $66.7 \%$ in the first two attempts to $1.3 \%$ in the third execution trial. It is also visible that from the three demonstrated sequences the task representation favors two specific serial orders.

Fig. 28 depicts the temporal evolution of $u^{p a}$ for the columns RC, GC and MC and for the TP during rehearsal. Figs. 28a and 28b correspond to the second and third rehearsal period, respectively. One can see that during the second rehearsal at time $t_{T P}$ when the placing of TP is simulated, the activities of the populations encoding MC and RC are above the learning threshold $\lambda^{p a}$ while the activity of the GC population is below. During the third rehearsal, however, the memory representations of all the three columns are above $\lambda^{p a}$ at time $t_{T P}$.

\subsection{Temporal differences between observation and internal simula- tion}

As already mentioned earlier, the time courses of population activity differ significantly during observational and off-line learning (Fig. 29). During rehearsal, the execution speed appears to be increased by a factor 5 which makes it possible to simulate the 30 rehearsals in less than $3 \mathrm{~min}$. The relatively short waiting time between successive demonstrations is an attractive feature for the observational learning approach. But perhaps more important, the variability in the inter-event intervals, which most likely will differ significantly between users and trials in natural learning situations, appears to be much reduced during task simulation. Since the memorized pattern in STM acts as stationary input to $u^{p r}$, the approximately constant duration of the transient population response in $u^{p r}$ defines the time interval between successive events. This reduced variability is a prerequisite for an adaptation mechanism that adjusts the time window for associative learning in response to negative feedback by the tutor. Since the inter-event intervals are predictable for the learning system, the time course of the persistent activity can be adjusted iteratively to include increasingly more past events in the learning process.

\section{Discussion and future work}

For decades, the idea has been discussed in animal and human research that off-line periods after learning such as awake resting or sleep promote the gradual incorporation of newly acquired information into long-term memory representations. Offline improvements without further practice have been reported in a wide variety of perceptual, motor and complex cognitive tasks (Stickgold, 2005). The mental replay of memory traces of recent behavioral experiences has been hypothesized to play an essential role in this consolidation process (Preston \& Eichenbaum, 2013).

In this paper, we have tested in real-world experiments with the humanoid robot ARoS a novel DNF model that implements the repeated reactivation of past experiences during off-line periods as a key mechanism to efficiently acquire the structure of an assembly plan from a small number of user demonstrations. The work complements our previous experimental studies with a DNF 
a

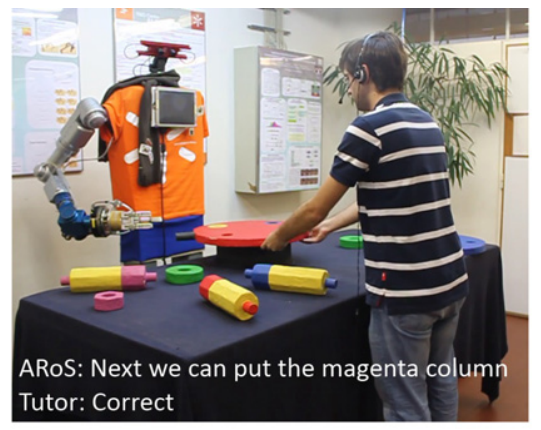

C

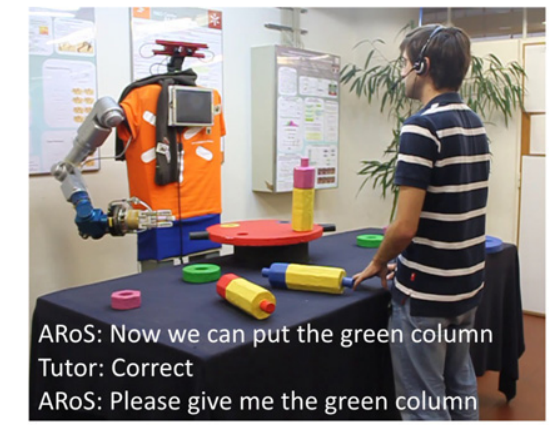

b

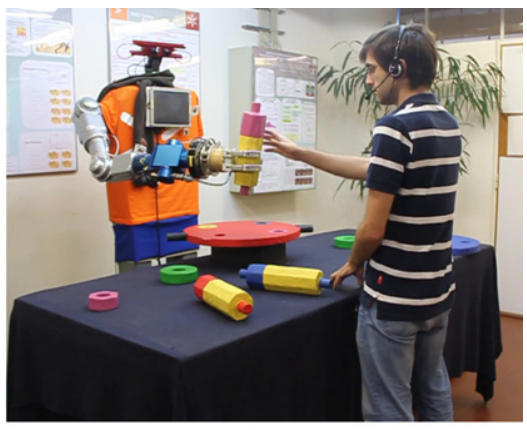

d

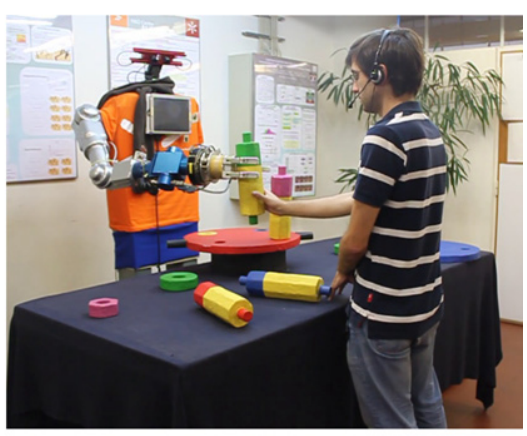

e

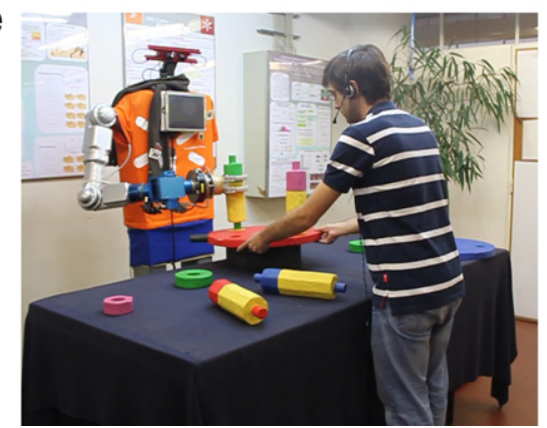

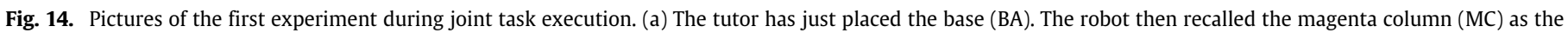

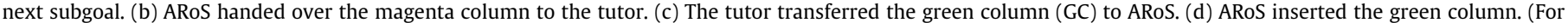
interpretation of the references to color in this figure legend, the reader is referred to the web version of this article.)

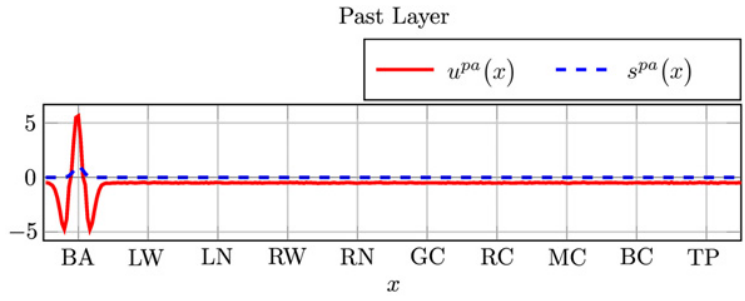

Present Layer

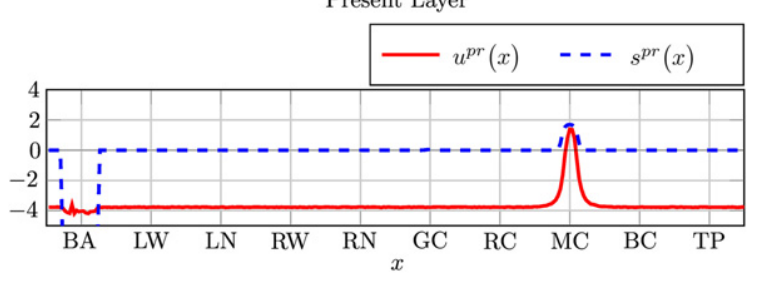

Fig. 15. First experiment. Snapshots of the activity pattern in the two LTM layers during execution are shown. With the base (BA) in place the model recalls the magenta column (MC) as the next subgoal.

architecture for natural human-robot interactions in which the shared task knowledge about the serial order of assembly steps was hand-coded by the designer (Bicho et al., 2011).

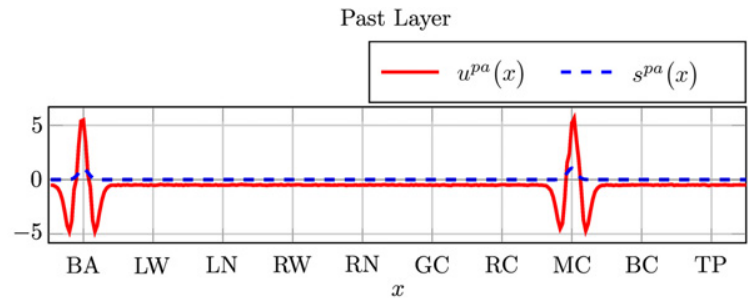

Present Layer

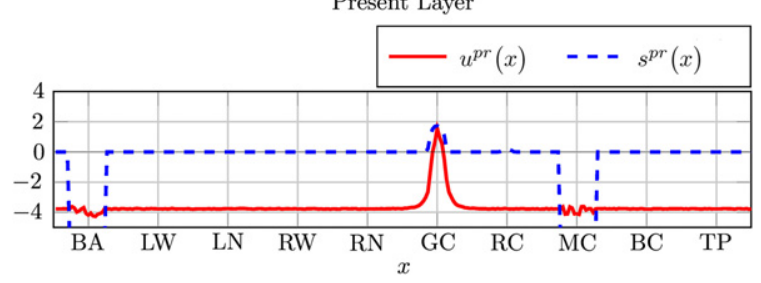

Fig. 16. First experiment. Snapshots of the activity pattern in the two LTM layers during execution are shown. With the base (BA) in place and the magenta column (MC) inserted, the model recalls the green column (GC) as the next subgoal.

The DNF implementation of efficient task learning is consistent with the principles of novel robotic designs in the field of neurorobotics. According to Krichmar (2008), key features of a 

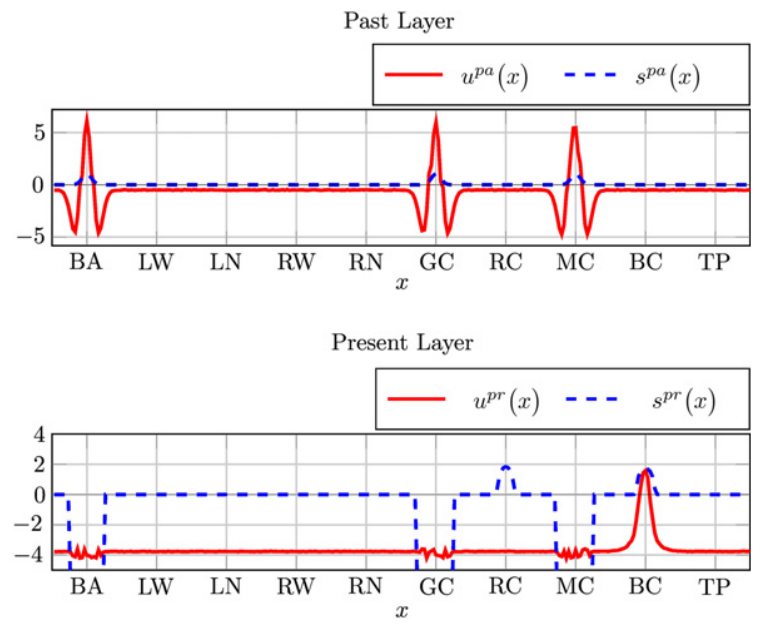

Fig. 17. Second experiment. Snapshots of the activity pattern in the two LTM layers during execution are shown. Following the placing of GC and MC, there is localized input to two populations, $\mathrm{RC}$ and $\mathrm{BC}$, in $u^{p r}$. Due to noise, the representation of $\mathrm{BC}$ won the competition in this trial.

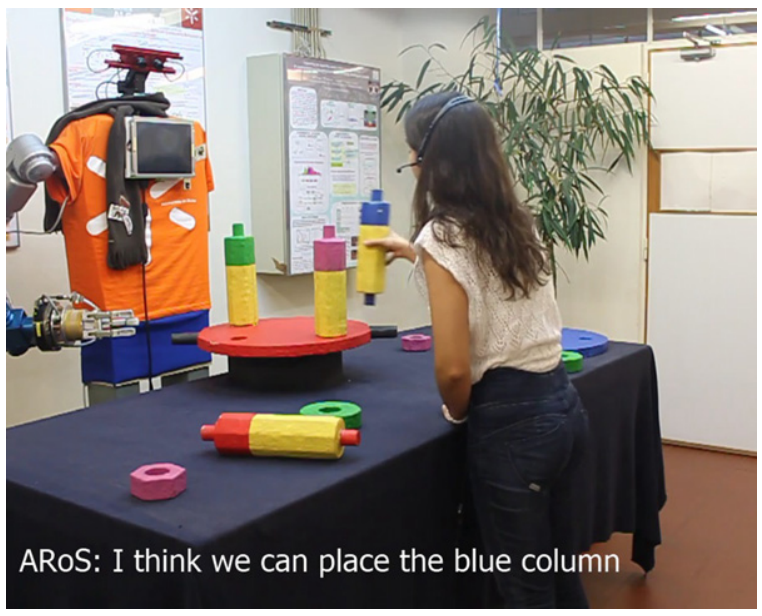

Fig. 18. Picture of the second experiment during joint execution. Both the green column (GC) and the magenta columns (MC) were already inserted. ARoS recalled the blue column BC to be placed next and the user executed this subtask. (For interpretation of the references to color in this figure legend, the reader is referred to the web version of this article.)

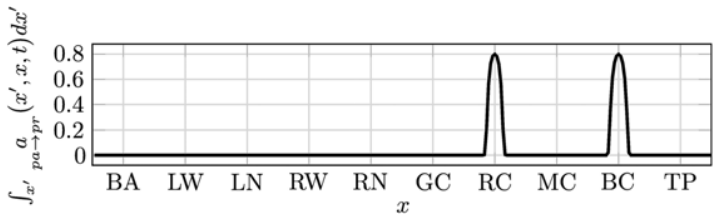

Fig. 19. The summed connection strengths of all newly established weights that link active populations in $u^{p a}$ to the two populations RC and BC in $u^{p r}$ are compared. The total input is approximately equal for both subgoals.

neurorobotic device are (1) a controller inspired by processing principles of the neural system, and (2) the development of new competences and skills through the interaction with a real-world environment. The architecture of the DNF model reflects converging lines of neurophysiological and computational evidence suggesting the existence of two complementary learning systems in the brain (McClelland et al., 1995; O'Reilly \& Norman, 2002). The model combines a fast, activation based learning in the STM module to robustly represent sequential information from single task demonstrations with a slower, weight-based learning in the LTM module to extract in off-line rehearsal periods
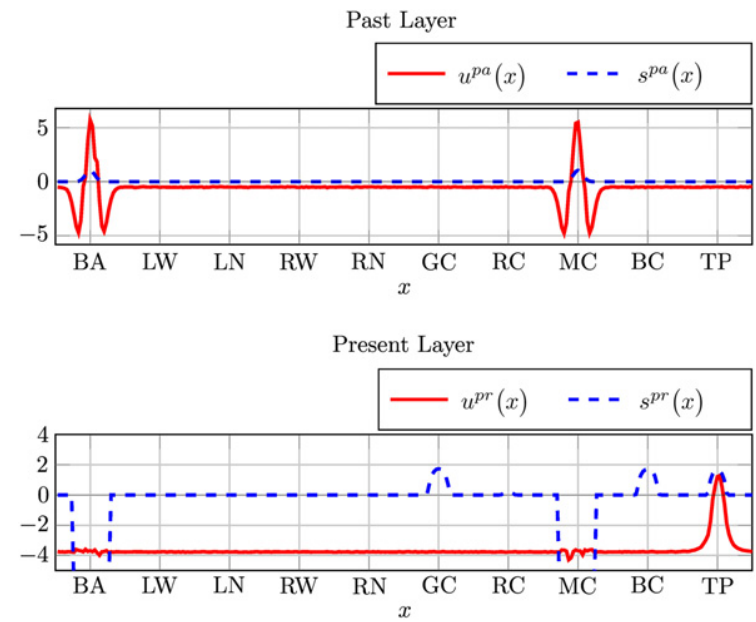

Fig. 20. Snapshot of activation patterns in LTM showing a prediction error. With BA and $\mathrm{MC}$ in place, the learned connections support GC, BC and TP as possible subgoals. In this trial, the top platform TP is prematurely chosen.

knowledge about possible orders and longer-term dependencies between subgoals. The theoretical framework of dynamic neural fields explains the emergence of self-sustained neural population activity which allows us to implement a neural learning dynamics on a behaviorally relevant time scale. ARoS acquires the task knowledge in real learning interactions with different human teachers. The real-world test of the theoretical model highlights specific challenges that could be easily overlooked in a pure modeling approach like for instance the variability in the duration of the task demonstrations (see the discussion below). The success of the robotics experiments show that the reactivation of a previously experienced serial order allows the learning system to incrementally incorporate new information in an existing memory structure. At the end of the learning process, ARoS knows that the columns can be inserted in any order. This allows the robot to react during joint task execution in a flexible manner to environmental constraints (e.g., a specific column is momentarily out of reach) or to adapt to an order preference that a human co-actor might show (Bicho et al., 2011). In addition, the sufficient and necessary condition for placing the top platform is represented by the parallel activation of all 4 columns in the past layer of LTM.

The benefits of the off-line improvement for the learningby-demonstration approach is obvious. It is simply unrealistic to assume that ordinary users would accept to supervise a large number of experimental trials or to re-demonstrate earlier sequences (Pardowitz et al., 2007). Since nearly all of our daily routine tasks are sequential in nature, the ability to learn new sequential orders through guidance by untrained users is a fundamental skill for a useful robot assistant. Beyond the specific application, exploring the possibility to endow robots with a human-like, off-line learning capacity offers new perspectives for many task domains (Stickgold, 2005).

To focus on the cognitive aspects of the learning task, we simplified in the experiments the initial perceptual acquisition of sequential information. The learning-by-demonstration approach requires in general high-level motor and perceptual competencies even if only action effects (e.g., changes in the to be assembled object) and not the continuous effector motions have to be imitated. The use of color and shape as the essential features that guides sequence learning is obviously not representative for most of our daily sequential activities. The present experiments share this limitation with earlier work in which mobile robots learned in a single demonstration to visit and manipulate objects in a specific, color-coded order (Nicolescu \& Matarić, 2003; Sandamirskaya \& Schöner, 2010). Moreover, the experimental setting was designed 

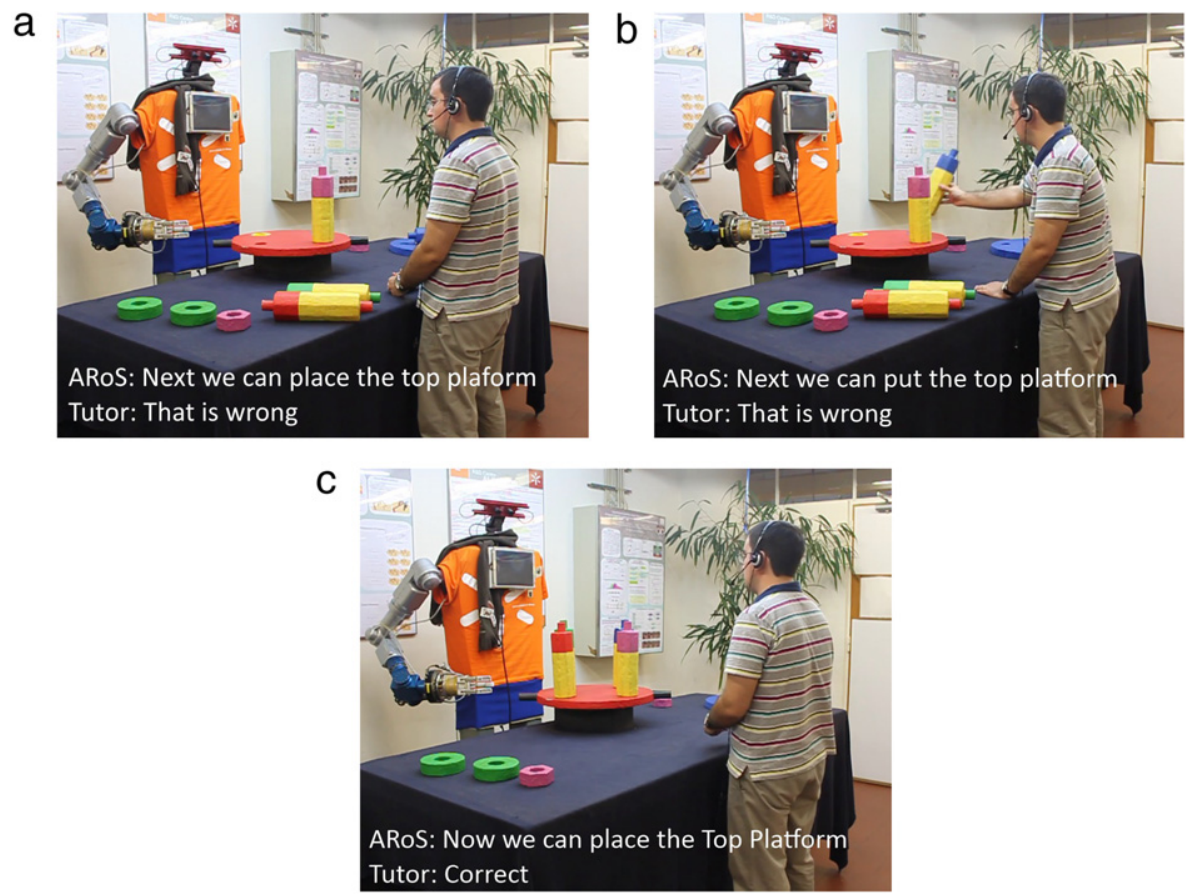

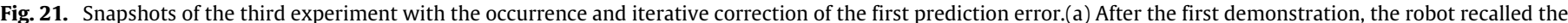

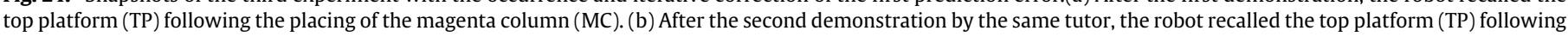

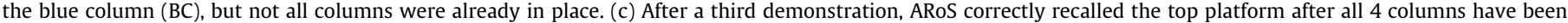
inserted. (For interpretation of the references to color in this figure legend, the reader is referred to the web version of this article.)

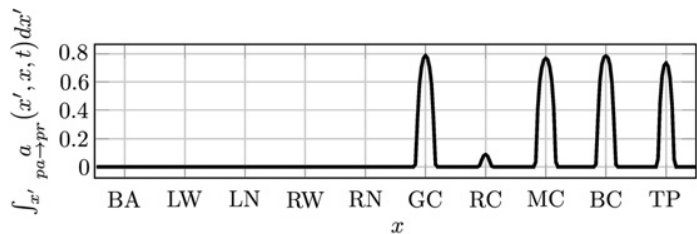

Fig. 22. Comparison of the summed connection strengths from active populations in $u^{p a}$ to populations GC, MC, BC and TP in $u^{p r}$. As can be seen, the total input to all four populations is very similar. Since MC had been already placed, this choice appears to be inhibited due to strong feedback inhibition (see Fig. 20).

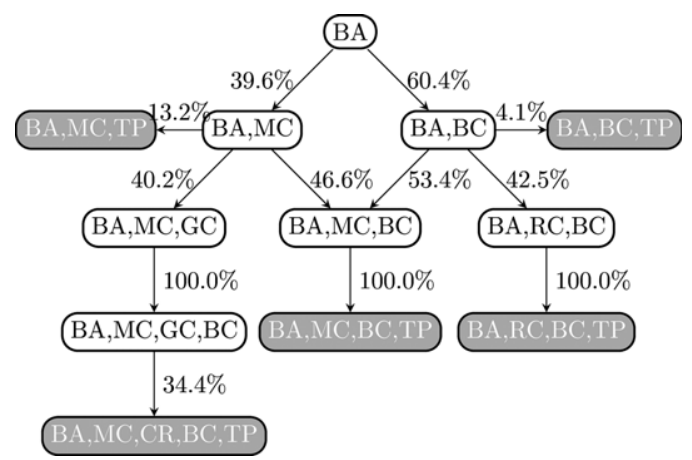

Fig. 23. Probability graph of the decision process in LTM following the first demonstration of the third sequence. The numbers indicate the transition probabilities to the next assembly state. Only the paths that lead to an error are represented. By summing the probabilities of each error, one can verify that the overall probability of an error occurrence is equal to $89.6 \%$.

to minimize the risk of occlusion. Some of the limitations may be overcome by integrating information about the users' objectdirected actions as in the goal inference capacity implemented in the DNF architecture for natural HRI (Bicho et al., 2011, 2010).

Towards the ultimate goal of efficient "one-shot" learning of task knowledge, several robotics experiments have explored the role of verbal interactions between user and robot in the
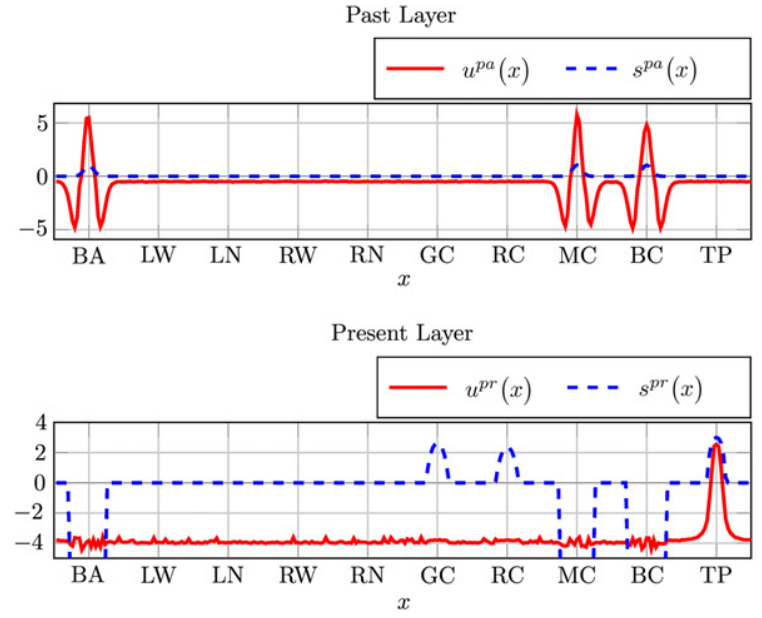

Fig. 24. Snapshots of the activation pattern in the two LTM layers illustrating the second error. With the base in place and both the magenta column (MC) and the blue column $(\mathrm{BC})$ inserted, the decision was taken again to place the top platform (TP).

learning by demonstration paradigm (Nicolescu \& Matarić, 2003; Pardowitz et al., 2007). Verbal feedback by the human user about intended or executed actions of the robot is considered an efficient and at the same time intuitive means to accelerate the learning process. The nature of the feedback may range from simple correct-wrong comments like in the present experiments to more sophisticated guidance like for instance high-lighting task irrelevant observations, explaining omission errors or describing the current behavior. A higher level of abstraction requires the implementation of sophisticated verbal communication skills and reasoning mechanisms in the robot which is typically achieved by employing formal logic and linguistic systems operating on symbols (Levesque \& Lakemeyer, 2008). While there is no doubt that these high-level competencies may greatly reduce the need 


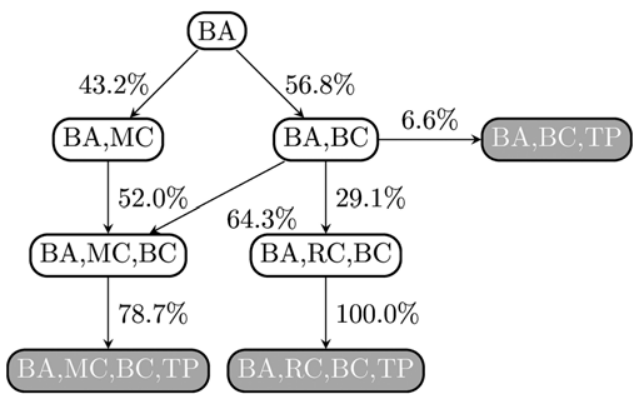

Fig. 25. Probability graph of the decision process in LTM following the second demonstration of the third sequence. After the second demonstration more past events affect the robot's prediction. The error probability of $66.7 \%$ remains nevertheless quite high.

for many overt (or covert) repetitions of the sequence during learning and practice it is also clear that an expert designer is needed to pre-structure the task-dependent learning algorithm with ordering constraints and the pre- and postcondition features of each subtask. Our neuro-inspired approach instead is based on a few general processing principles like stable activation patterns induced by transient inputs, an adaptive learning threshold and internal simulations of past experiences that allow ARoS to autonomously develop representations of the task rules in a social learning situation.

A neurorobotic model may not only serve to develop more effective robots but may also provide an embodied test bed for theories of brain function (Krichmar, 2008). In order to further refine the functional two-stage learning model, we plan in future work to compare model assumptions and specific aspects of its dynamic behavior with findings in human sequence learning studies. For the present implementation, the STM module stored only the last demonstration. It is however straightforward to encode several independent representations of serial order in a STM with several fields. A neural correlate for such a memory storage is not completely clear however. Neural activity has been reported in various brain areas that exhibits selectivity to specific temporal orders of the occurrence of visual cues when the sequence information was important to execute an associated multi-step behavior in the same order after a delay (Ninokura, Mushiake, \& Tanji, 2003; Tanji, 2001). The re-activation of not just one but multiple short-term memories during off-line periods might have an important impact on the developing memory structure in LTM. Findings in behavioral studies suggest for instance that longer sequences are acquired through the concatenation of shorter chunks during random interleaved practice, but that during blocked practice concatenation does not occur. In general, the type of practice seem to affect the stability of the memory trace (Robertson, Pascual-Leone, \& Miall, 2004). It would be highly interesting to extend the off-line learning model with the capacity to randomly select from multiple examples in STM in order to systematically test the impact of an interleaved versus a blocked design on the structure and stability of LTM representations.

In line with our findings, neuro-plastic processes during off-line periods following practice are believed to support not only the ongoing process of memory consolidation but also the discovery of an underlying structure or rule governing the sequential events (Stickgold, 2005). Employing a serial reaction time task (SRTT) paradigm, Fischer and colleagues (Fischer, Drosopoulos, Tsen, \& Born, 2006) have shown for instance that sleep greatly facilitates the gain of explicit knowledge of a hidden rule performed under "implicit" learning conditions before sleep. In the SRTT paradigm, subjects respond as fast and as accurately as possible to a sequence of a target stimulus on a screen with
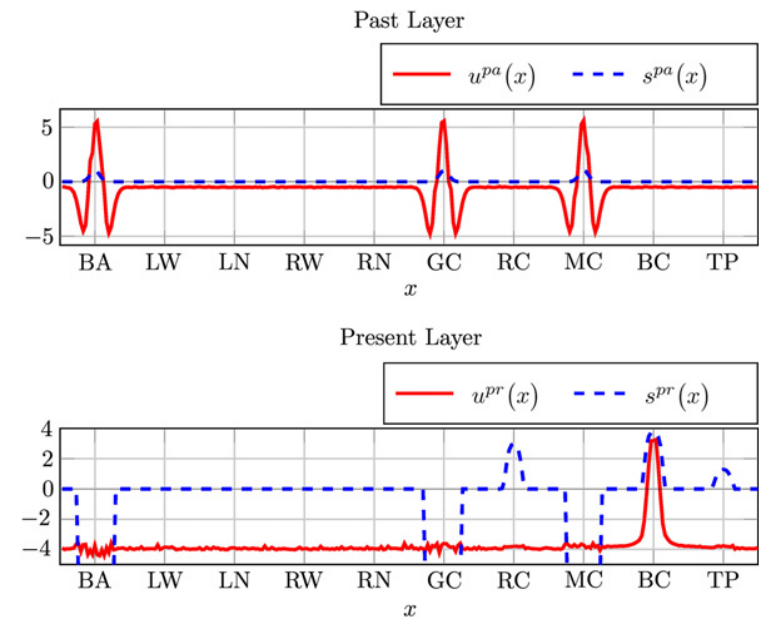

Fig. 26. Activation patterns in LTM after the iterative error corrections. Following the third demonstration, the active populations BA, GC and MC in $u^{p a}$ support the correct prediction to select $\mathrm{BC}$ as the next subtask.

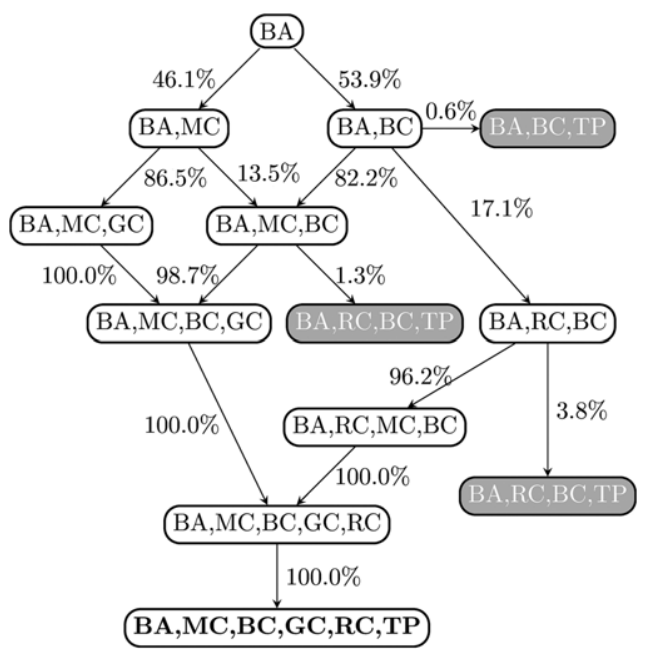

Fig. 27. Probability graph of the decision process in LTM following the third repetition. The time window for associative learning is now long enough to represent the sequential dependencies between the four columns and the TP. As a consequence, the error probability dropped to $1.3 \%$.

a spatially corresponding key. Learning is implicit since subjects do not express awareness of a repeating pattern in the sequence despite a significant reduction in reaction time (RT) compared to a random sequence. Although the DNF model does not integrate any specific sleep-dependent mechanisms, it is tempting to speculate that the transition from implicit to explicit knowledge occurs when the newly establish connection in LTM are sufficiently strong to predict the identity of the forthcoming subgoal. In the model, this prediction is associated with the evolution of a self-stabilized bump. In an early learning phase, the input from the past layer is relatively weak and only preshapes the neural population representation of the next assembly step. This priming mechanism nevertheless facilitates the processing of the visual stimulus signaling the accomplishment of the subgoal (Erlhagen \& Schöner, 2002).

In LTM, the time course of persistent population activity above a learning threshold (Seitz \& Dinse, 2007) defines the time window for establishing associations between successive events in the two DNF layers. The concept of a specific time window for a long-term memory formation on a behaviorally relevant time scale is consistent with recent findings showing that only the initial phase of 
a

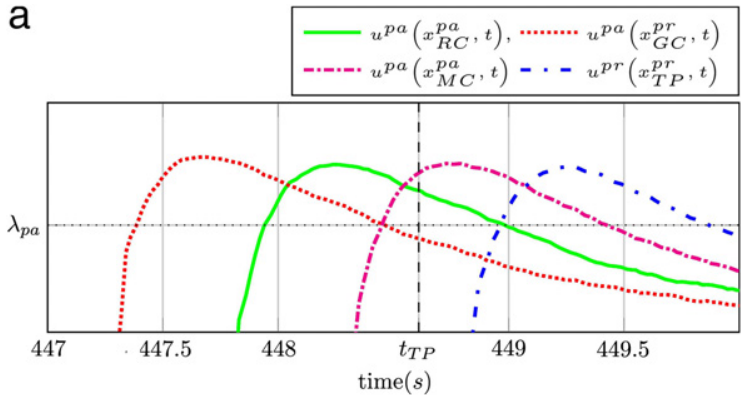

b

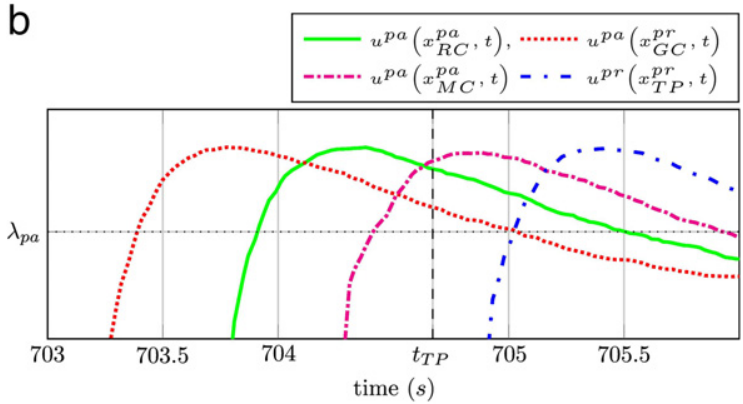

Fig. 28. Temporal evolution of population activities in $u^{p a}$ during the second (a) and third (b) rehearsal period of the third experiment. (a) After the first error, the decay rate of $h_{p a}$ decreased. When the placing of TP is simulated at time $t_{T P}$ in $u^{p r}$, the activities of the populations representing MC and RC in $u^{p a}$ are above the learning threshold. (b) After the second error, the decay rate of $h_{p a}$ is further decreased. Now, also the population representation of GC in $u^{p a}$ is above threshold at time $t_{T P}$.

working memory maintenance actually contributes to LTM formation (Ranganath, Cohen, \& Brozinsky, 2005). The model assumption that this time window may vary with specific demands of the learning task (e.g., error correction) could be tested in behavioral and neurophysiological experiments. It is known that the strength and the time course of persistent activity in prefrontal areas may be modulated by actions and their outcomes across multiple trials (Curtis \& Lee, 2010). We use this idea to increase the duration of population activity above the (fixed) learning threshold whenever the robot makes a prediction error about the next subgoal. In future work, it would be interesting to explore other possible mechanisms like for instance a long-term modulation of the learning threshold as well that has been suggested as a neuro-plausible mechanism for adaptive event timing (Ivry \& Spencer, 2004). Independent of the specific implementation, the adaptive time window mechanism only works since the replay reflects the system's intrinsic dynamics when not constrained by the time course of external input stimuli. The constant but accelerated speed of the covert sequence rehearsals solves the challenging real-time constraints of a learning-by-demonstration approach in which the execution duration may vary significantly between trials and users. Interestingly, the observed time-compressed reactivation of neural activity patterns in Hippocampus have been taken as evidence that the sequential structure of the behavioral experience, rather than the detailed time course of particular episodes is replayed (Nádasdy, Hirase, Czurkó, Csicsvari, \& Buzsáki, 1999).

Following our dynamic neural field approach towards natural human-robot interactions, we have taken inspiration from processing principles of biological cognitive systems to develop an artificial cognitive system able to process, re-structure and consolidate memories off-line. The assembly task example allowed us to illustrate the DNF implementation of two complementary learning systems under real-world constraints. It is clear that the concept of "sleeping on a problem" for robots remains still a far fetched goal. We plan to improve and test the dynamic field model along the lines just discussed.

\section{Acknowledgments}

The work was funded by FCT - Fundação para a Ciência e Tecnologia, through the PhD Grants SFRH/BD/48529/ 2008 and SFRH/BD/41179/2007 and Project NETT: Neural Engineering Transformative Technologies, EU-FP7 ITN (nr.289146) and the FCTResearch Center CMAT (PEst-OE/MAT/UI0013/ 2014).

\section{Appendix A. Mathematical formulation of STM module}

Input to $u^{\text {stm }}$. The field $u^{\text {stm }}$ receives input only from the vision system given by

$s^{s t m}(x, t)=C^{s t m} s^{v i}(x, t)$,

where the gain parameter $C^{\text {stm }}$ controls the input strength.

Resting state adaptation in $u^{\text {stm }}$. The adaptation dynamics of the resting state $h^{\text {stm }}$ of excited populations establishes the activation gradient in the STM field (for details see Ferreira et al., 2014). It is governed by the following equation:

$$
\begin{aligned}
\tau_{h^{s t m}} \frac{\partial h^{s t m}(x, t)}{\partial t}= & \left(1-f_{0}\left[u^{s t m}(x, t)\right]\right)\left(-h^{s t m}(x, t)\right. \\
& \left.+H_{i n i}^{s t m}\right)+f_{0}\left[u^{s t m}(x, t)\right],
\end{aligned}
$$

where $H_{i n i}^{s t m}$ is the initial value of $h^{\text {stm }}$ and $\tau_{h^{s t m}}$ controls the growth rate of $h^{\text {stm }}$. When $u^{\text {stm }}<0$, the resting level $h^{\text {stm }}$ simply converges to the initial value $H_{i n i}^{s t m}$. If $u^{s t m}>0$, the derivative is equal to one and $h^{\text {stm }}$ grows linearly. In the robotics experiments, the growth is stopped when the tutor issues the sentence "I have finished".
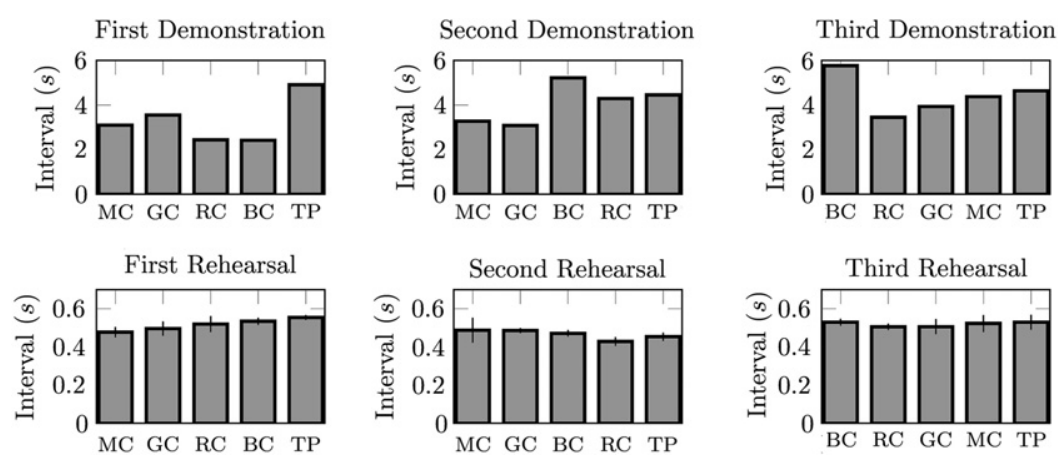

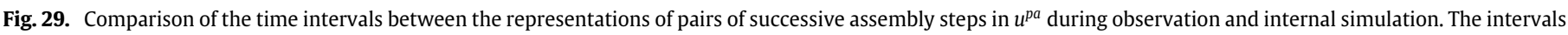

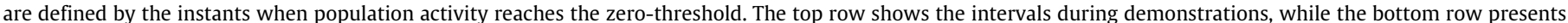
the average (bar) and standard deviation (error) of the rehearsals. Note the different time scales for the figures in the two rows. 


\section{Appendix B. Mathematical formulation of LTM module}

Input to $u^{p a}$ and $u^{p r}$. The input $s^{p a}$ to field $u^{p a}$ is given by

$$
\begin{aligned}
s^{p a}(x, t)= & C^{p a} s^{v i}(x, t) \\
& +\int f_{\lambda p r}\left[u^{p r}(x, t)\right] \underset{p r \rightarrow p a}{E}\left(x^{\prime}, x\right) d x^{\prime},
\end{aligned}
$$

where $s^{v i}$ is the input from the vision system and the integral term represents the summed input from $u^{p r}$ mediated by pre-defined excitatory connections $\underset{p r \rightarrow p a}{E}$ between corresponding populations. The threshold function $f_{\lambda p r}[\cdot]$ ensures that only activity above $\lambda^{p r}$ is propagated.

For the field $u^{p r}$, the input $s^{p r}$ is given by

$$
\begin{aligned}
s^{p r}(x, t)= & C^{p r} s^{v i}(x, t) \\
& +b^{r e a} C^{r e a} u^{s t m}(x, t) \\
& +\int f_{0}\left[u^{p a}\left(x^{\prime}, t\right)\right]_{p a \rightarrow p r}^{I}\left(x^{\prime}, x\right) d x^{\prime} \\
& +\int f_{0}\left[u^{p a}\left(x^{\prime}, t\right)\right] \underset{p a \rightarrow p r}{a}\left(x^{\prime}, x, t\right) d x^{\prime}
\end{aligned}
$$

which includes the vision input, the input from STM applied during rehearsal by choosing $b^{\text {rea }}=1$, the propagation of activity $u^{\text {pa }}$ through the pre-defined inhibitory connections $\underset{p a \rightarrow p r}{I}$, and the adaptive excitatory connections $\underset{p a \rightarrow p r}{a}$ that are established during the learning process. The Heaviside step function $f_{0}[\cdot]$ (see Eq. (2)) is applied to guarantee that activity propagates from $u^{p a}$ to $u^{p r}$ only when it is suprathreshold.

Adaptation dynamics for the resting state. The dynamics in $u^{p a}$ is coupled with an adaptation dynamics for the resting state $h^{p a}$ of excited populations (for a discussion of a mathematically equivalent threshold accommodation dynamics for the transfer function see Coombes \& Owen, 2007). This adaptation dynamics is used to control the time period during which the population activity is above the learning threshold $\lambda^{p a}$. It is governed by the following differential equation

$$
\begin{aligned}
\tau_{h^{p a}} \frac{\partial h^{p a}(x, t)}{\partial t}= & \left(1-f_{0}\left[u^{p a}(x, t)\right]\right)\left(h_{b a s}^{p a}-h^{p a}(x, t)\right) \\
& +f_{0}\left[u^{p a}(x, t)\right]\left(H_{d e c}^{p a}-h^{p a}(x, t)\right),
\end{aligned}
$$

where $\tau_{h p a}$ defines the adaptation rate. The value of $h^{p a}$ will tend to the baseline value $h_{\text {bas }}^{\text {pa }}$ when there is no suprathreshold activity in the field and decays to $H_{d e c}^{p a}<h_{b a s}^{p a}$ for field regions with suprathreshold activity. The baseline value $h_{\text {bas }}^{\text {pa }}$ may vary between two values depending on whether the robot is in the learning or in the execution phase of the experiments. During internal rehearsal the value is chosen as $h_{\text {bas }}^{p a}=H_{\text {low }}^{p a}$ whereas during active recall the value is higher $h_{\text {bas }}^{\text {pa }}=H_{\text {high }}^{p a}$.

\section{Appendix C. Implementation parameters}

See Tables C.1-C.4.

\section{Appendix D. Supplementary data}

Supplementary material related to this article can be found online at http://dx.doi.org/10.1016/j.neunet.2015.09.002.
Table C.1

Implementation parameters of the Short Term Memory (STM) module.

\begin{tabular}{lll}
\hline$\tau^{s t m}$ & Time constant of $u^{s t m}$ & 3.0 \\
$A^{s t m}$ & Kernel amplitude & 2.5 \\
$b^{s t m}$ & $b$ parameter of the kernel & 0.3 \\
$\alpha^{s t m}$ & $\alpha$ parameter of the kernel & 0.15 \\
$C^{s t m}$ & Gain parameter of visual input to $u^{s t m}$ & 7.0 \\
$\zeta_{s t o c h}^{s t m}$ & Amplitude of Gaussian $[N(0,1)]$ noise in $u^{s t m}$ & 0.5 \\
$\tau_{h^{s t m}}^{s t m}$ & Time constant of the $h^{s t m}$ variation & 0.2 \\
$H_{i n i}^{s t m}$ & Initial value of the resting level & -4.0 \\
\hline
\end{tabular}

Table C.2

Implementation parameters of the past layer $u^{\text {pa }}$ of the Long Term Memory (LTM) module.

\begin{tabular}{lll}
\hline$\tau^{p a}$ & Time constant of $u^{p a}$ & 2.0 \\
$A^{p a}$ & Kernel amplitude & 3.2 \\
$b^{p a}$ & $b$ parameter of the kernel & 0.35 \\
$\alpha^{p a}$ & $\alpha$ parameter of the kernel & 0.5 \\
$H_{l o w}^{p a}$ & Lower baseline value of $h^{p a}$ & -1.0 \\
$H_{h i g h}^{p a}$ & Higher baseline value of $h^{p a}$ & -0.5 \\
$H_{d e c}^{p a}$ & Resting value of $h^{p a}$ & -4.5 \\
$C^{p a}$ & Gain parameter of visual input to $u^{p a}$ & 1.05 \\
$\zeta_{\text {stoch }}^{p a}$ & Amplitude of Gaussian $[N(0,1)]$ noise in $u^{p a}$ & 0.1 \\
$\lambda^{p a}$ & Learning threshold of $u^{p a}$ & 5.3 \\
$T_{1}$ & Time constant of the $h^{p a}$ decay with no error & 20.0 \\
$T_{2}$ & Time constant of the $h^{p a}$ decay - one error & 35.0 \\
$T_{3}$ & Time constant of the $h^{p a}$ decay - two errors & 56.0
\end{tabular}

Table C.3

Implementation parameters of the present layer $u^{p r}$ of the Long Term Memory (LTM) module.

\begin{tabular}{llr}
\hline$A^{p r}$ & Kernel amplitude & 9.4 \\
$\sigma^{p r}$ & Standard deviation of the kernel & 7.0 \\
$w_{\text {inhib }}^{p r}$ & Global inhibition of the kernel & 8.8 \\
$\tau^{p r}$ & Time constant of $u^{p r}$ & 2.0 \\
$H^{p r}$ & Value of the resting level & -1.6 \\
$\lambda^{p r}$ & Learning threshold of $u^{p r}$ & 5.0 \\
$C^{p r}$ & Gain parameter of visual input to $u^{p r}$ & 17 \\
$\zeta_{\text {stoch }}^{p r}$ & Amplitude of Gaussian $[N(0,1)]$ noise in $u^{p r}$ & 2.0 \\
$C^{r e a}$ & Excitation from $u^{\text {stm }}$ during rehearsal & 0.9 \\
\hline
\end{tabular}

Table C.4

Implementation parameters of the learning rule.

\begin{tabular}{lll}
\hline$\tau_{a}$ & Time constant of the learning rule & 4.0 \\
$\eta$ & Parameter of the decay term of the learning rule & 0.1 \\
\hline
\end{tabular}

\section{References}

Amari, S.-i. (1977). Dynamic of pattern formation in lateral inhibition type neural fields. Biological Cybernetics, 27, 77-87.

Bandura, A. (1971). Social learning theory. Prentice-Hall: Englewood Cliffs.

Bicho, E., Erlhagen, W., Louro, L., \& Costa e Silva, E. (2011). Neuro-cognitive mechanisms of decision making in joint action: a human-robot interaction study. Human Movement Science, 30(5), 846-868.

Bicho, E., Louro, L., \& Erlhagen, W. (2010). Integrating verbal and nonverbal communication in a dynamic neural field architecture for human-robot interaction. Frontiers in Neurorobotics, 4, 135-164.

Billard, A. G., Calinon, S., Dillmann, R., \& Schaal, S. (2008). Robot programming by demonstration. In B. Siciliano, \& O. Khatib (Eds.), Handbook of Robotics (pp. 1371-1394). Secaucus, NJ, USA: Springer.

Cleeremans, A., \& McClelland, J. L. (1991). Learning the structure of event sequences. Journal of Experimental Psychology. General, 120(3), 235-253.

Coombes, S., \& Owen, M. (2007). Exotic dynamics in a firing rate model of neural tissue with threshold accommodation. In AMS Cont. Math (pp. 123-144).

Curtis, C. E., \& Lee, D. (2010). Beyond working memory: The role of persistent activity in decision making. Trends in Cognitive Sciences, 14(5), 216-222.

Dautenhahn, K., \& Nehaniv, C. L. (2002). Imitation in animals and artifacts. Cambridge, MA, USA: MIT Press.

Elman, J. L. (1990). Finding structure in time. Cognitive Science, 14, 179-211.

Erlhagen, W., \& Bicho, E. (2006). The dynamic neural field approach to cognitive robotics. Journal of Neural Engineering, 3(3), R36-R54.

Erlhagen, W., \& Schöner, G. (2002). Dynamic field theory of movement preparation. Psychological Review, 109(3), 545-572. 
Euston, D. R., Tatsuno, M., \& McNaughton, B. L. (2007). Fast-forward playback of recent memory sequences in prefrontal cortex during sleep. Science, 318(5853), 1147-1150.

Ferreira, F., Erlhagen, W., Sousa, E., \& Bicho, E. (2014). Learning a musical sequence by observation: A robotics implementation of a dynamic neural field model In Development and learning and epigenetic robotics (ICDL-Epirob), 2014 joint IEEE international conferences on (pp. 157-162). Genova, Italy: IEEE.

Fischer, S., Drosopoulos, S., Tsen, J., \& Born, J. (2006). Implicit learning - explicit knowing: a role for sleep in memory system interaction. Journal of Cognitive Neuroscience, 18(3), 311-319.

Genovesio, A., Brasted, P. J., \& Wise, S. P. (2006). Representation of future and previous spatial goals by separate neural populations in prefrontal cortex. The Journal of Neuroscience, 26(27), 7305-7316.

Grossberg, S. (1978). Behavioral contrast in short term memory: Serial binary memory models or parallel continuous memory models? Journal of Mathematical Psychology, 17(3), 199-219.

Ikeuchi, K., \& Suehiro, T. (1994). Toward an assembly plan from observation. I. Task recognition with polyhedral objects. IEEE Transactions on Robotics and Automation, 10, 368-385.

Ivry, R. B., \& Spencer, R. M. C. (2004). The neural representation of time. Current Opinion in Neurobiology, 14, 225-232.

Krichmar, J. (2008). Neurorobotics. Scholarpedia, 3(3), 1365.

Laing, C. R., Troy, W. C., Gutkin, B., \& Ermentrout, G. B. (2002). Multiple bumps in a neuronal model of working memory. SIAM Journal on Applied Mathematics, 62(1), 62-97

Levesque, H., \& Lakemeyer, G. (2008). Cognitive robotics. In Foundations of artificial intelligence, vol. 3 (pp. 869-886). Ch. 23.

McClelland, J. L., O'reilly, R. C., \& McNaughton, B. L. (1995). Why there are complementary learning systems in the hippocampus and neocortex: insights from the successes and failures of connectionist models of learning and memory. Psychological Review, 102(3), 419-457.

Miller, E. K. (2000). The prefrontal cortex and cognitive control. Nature reviews Neuroscience, 1, 59-65.

Nádasdy, Z., Hirase, H., Czurkó, A., Csicsvari, J., \& Buzsáki, G. (1999). Replay and time compression of recurring spike sequences in the hippocampus. The Journal of Neuroscience, 19(21), 9497-9507.

Nicolescu, M. N., \& Matarić, M. J. (2003). Natural methods for robot task learning: instructive demonstrations, generalization and practice. In AAMAS'03: Proceedings of the second international joint conference on Autonomous agents and multiagent systems (pp. 241-248). New York, NY, USA: ACM.

Ninokura, Y., Mushiake, H., \& Tanji, J. (2003). Representation of the temporal order of visual objects in the primate lateral prefrontal cortex. Journal of Neurophysiology, 89(5), 2868-2873.

O’Reilly, R. C., \& Norman, K. a. (2002). Hippocampal and neocortical contributions to memory: Advances in the complementary learning systems framework. Trends in Cognitive Sciences, 6(12), 505-510.

Otero, N., Saunders, J., Dautenhahn, K., \& Nehaniv, C. L. (2008). Teaching robot companions: The role of scaffolding and event structuring. Connection Science, $20,111-134$.
Pardowitz, M., Knoop, S., Dillmann, R., \& Zöllner, R. (2007). Incremental learning of tasks from user demonstrations, past experiences, and vocal comments. IEEE Transactions on Systems, Man and Cybernetics, Part B (Cybernetics), 37(2), 322-332.

Preston, A. R., \& Eichenbaum, H. (2013). Interplay of hippocampus and prefrontal cortex in memory. Current Biology, 23(17), R764-R773.

Ranganath, C., Cohen, M. X., \& Brozinsky, C. J. (2005). Working memory maintenance contributes to long-term memory formation: Neural and behavioral evidence. Journal of Cognitive Neuroscience, 17(7), 994-1010.

Rhodes, B. J., Bullock, D., Verwey, W. B., Averbeck, B. B., \& Page, M. (2004). Learning and production of movement sequences: Behavioral, neurophysiological, and modeling perspectives. Human Movement Science, 23(5), 699-746.

Robertson, E. M., Pascual-Leone, A., \& Miall, R. C. (2004). Current concepts in procedural consolidation. Nature reviews. Neuroscience, 5(July), 576-582.

Salinas, E. (2003). Background synaptic activity as a switch between dynamical states in a network. Neural Computation, 15(7), 1439-1475.

Sandamirskaya, Y., \& Schöner, G. (2010). An embodied account of serial order: How instabilities drive sequence generation. Neural Networks, 23, 1164-1179.

Schaal, S. (2007). The New Robotics-towards human-centered machines. HFSP journal, 1(January), 115-126.

Schöner, G. (2008). Dynamical systems approaches to cognition. In Cambridge handbook of computational cognitive modeling (pp. 101-126).

Seitz, A. R., \& Dinse, H. R. (2007). A common framework for perceptual learning. Current opinion in neurobiology, 17(2), 148-153.

Silva, R. M., Bicho, E., \& Erlhagen, W. (2008). Aros: An anthropomorphic robot for human-robot interaction and coordination studies. In Proceedings of the CONTROLO2008 Conference 8th Portuguese Conference on Automatic Control (pp. 819-826). Vila Real, Portugal: UTAD.

Silva, E. C., Costa, M. F., Araújo, J. P., Machado, D., Louro, L., Erlhagen, W., \& Bicho, E. (2014). Towards human-like bimanual movements in anthropomorphic robots: a nonlinear optimization. Applied Mathematics E' Information Sciences, 9(2), 1-10.

Stickgold, R. (2005). Sleep-dependent memory consolidation. Nature, 437(October), $1272-1278$.

Sutherland, G. R., \& McNaughton, B. (2000). Memory trace reactivation in hippocampal and neocortical neuronal ensembles. Current Opinion in Neurobiology, $10,180-186$

Tanji, J. (2001). Sequential organization of multiple movements: Involvement of cortical motor areas. Annual Review of Neuroscience, 24(July 2015), 631-651.

Thomaz, A. L., \& Breazeal, C. (2008). Teachable robots: Understanding human teaching behavior to build more effective robot learners. Artificial Intelligence, 172(6-7), 716-737.

Wardak, C., Ramanoël, S., Guipponi, O., Boulinguez, P., \& Ben Hamed, S. B. (2012). Proactive inhibitory control varies with task context. European Journal of Neuroscience, 36, 3568-3579.

Widrow, B., \& Hoff, M. E. (1960). Adaptive switching circuits. IRE WESCON Convention Record, New York: IRE, (Part 4), 96-104.

Wu, Y., \& Demiris, Y. (2010). Towards one shot learning by imitation for humanoid robots. In Robotics and Automation (ICRA), 2010 IEEE International Conference on (pp. 2889-2894). 